\title{
FOXP1 controls mesenchymal stem cell commitment and senescence during skeletal aging
}

\author{
Hanjun Li, ${ }^{1}$ Pei Liu, ${ }^{1}$ Shuqin Xu, ${ }^{1}$ Yinghua Li, ${ }^{2}$ Joseph D. Dekker, ${ }^{3}$ Baojie Li, ${ }^{1}$ Ying Fan, ${ }^{4}$ Zhenlin Zhang, ${ }^{4}$ Yang Hong, ${ }^{2}$ Gong Yang, ${ }^{2}$ \\ Tingting Tang, ${ }^{5}$ Yongxin Ren, ${ }^{6}$ Haley O. Tucker, ${ }^{3}$ Zhengju Yao, ${ }^{1}$ and Xizhi Guo ${ }^{1}$ \\ 'Bio-X-Renji Hospital Research Center, Renji Hospital, School of Medicine, Shanghai Jiao Tong University, Shanghai, China. '2Central Laboratory, The Fifth People's Hospital of Shanghai, Fudan University, \\ Shanghai, China. Institute for Cellular and Molecular Biology, University of Texas at Austin, Austin, Texas, USA. ${ }^{3}$ The Sixth People's Hospital of Shanghai, and ${ }^{5}$ The Ninth People's Hospital of Shanghai, \\ Shanghai Jiao Tong University, Shanghai, China. ${ }^{6}$ Department of Orthopaedics, The First Hospital, Nanjing Medical University, Nanjing, Jiangsu, China.
}

\begin{abstract}
A hallmark of aged mesenchymal stem/progenitor cells (MSCs) in bone marrow is the pivot of differentiation potency from osteoblast to adipocyte coupled with a decrease in self-renewal capacity. However, how these cellular events are orchestrated in the aging progress is not fully understood. In this study, we have used molecular and genetic approaches to investigate the role of forkhead box P1 (FOXP1) in transcriptional control of MSC senescence. In bone marrow MSCs, FOXP1 expression levels declined with age in an inverse manner with those of the senescence marker $p 16^{\text {INK } 4 A}$. Conditional depletion of Foxp 1 in bone marrow MSCs led to premature aging characteristics, including increased bone marrow adiposity, decreased bone mass, and impaired MSC selfrenewal capacity in mice. At the molecular level, FOXP1 regulated cell-fate choice of MSCs through interactions with the CEBP $\beta / \delta$ complex and recombination signal binding protein for immunoglobulin $\kappa$ J region (RBPјк), key modulators of adipogenesis and osteogenesis, respectively. Loss of p16 INK4A in Foxp1-deficient MSCs partially rescued the defects in replication capacity and bone mass accrual. Promoter occupancy analyses revealed that FOXP1 directly represses transcription of $p 16^{\text {INK4A }}$. These results indicate that FOXP1 attenuates MSC senescence by orchestrating their cell-fate switch while maintaining their replicative capacity in a dose- and age-dependent manner.
\end{abstract}

\section{Introduction}

Bone aging, the main risk factor for primary osteoporosis, results in a decrease in bone mass and a parallel increase of BM adiposity (1-3). At the cellular level, BM mesenchymal stem/progenitor cells (MSCs), which are the common progenitors from which osteoblasts and adipocytes arise, undergo senescence along with bone aging (4). Anatomically, MSCs, defined as PDGF- $\alpha^{+}$Sca $1^{+} \mathrm{CD} 31^{-} \mathrm{CD} 45^{-}$, reside primarily within the perivasculature - a region that can be specifically targeted by nestin-Cre (5), Prx1-Cre (6), or Lepr-Cre (7). As compared with young cells, senescent MSCs have reduced self-renewal capacity and predominantly differentiate into adipocytes as opposed to osteoblasts $(8,9)$. Such osteogenic or adipogenic commitment of MSCs is controlled by several crucial transcription factors. For example, PPAR $\gamma$ and $\mathrm{CEBP} \alpha / \beta / \delta$ constitute an essential cascade for the adipocyte program, whereas RUNX2 and osterix are master regulators of osteoblast differentiation (10-12).

Cellular senescence, a state of irreversible growth arrest, is mainly controlled by the $\mathrm{p} 16^{\mathrm{INK} 4 \mathrm{~A}} / \mathrm{Rb}$ and $\mathrm{p} 19^{\mathrm{ARF}} / \mathrm{p} 53$ pathways $(13$, 14). $\mathrm{p} 16^{\mathrm{INK} 4 \mathrm{~A}}$ is required for the age-associated decline in function of a number of adult stem cells (15-18), including BM MSCs (19). p53-Deficient mice display osteoporosis and other aging-related features (20). Alterations in the transcriptional control or activity of these pathways account for the aging-related features detected in mice deficient for Bmi-1 (21), Brac1 (22), Zmpste24 (23), Hmga2

Conflict of interest: The authors have declared that no conflict of interest exists

Submitted: July 11, 2016; Accepted: January 5, 2017.

Reference information: J Clin Invest. 2017;127(4):1241-1253.

https://doi.org/10.1172/JCI89511.
(24), or BUBR1 (25). In addition, Rb determines cell-fate choice and prevents premature aging of $\operatorname{MSCs}(26,27)$. However, the molecular network orchestrating adipo-osteogenic balance and cellular senescence of MSCs remains largely unknown.

Forkhead box P1 (FOXP1) is a transcriptional factor that controls multiple cell-differentiation pathways, including embryonic stem cell pluripotency (28), T and B cell development (29-31), lung epithelial cell-fate determination (32-35), cardiomyocyte proliferation $(33,36)$, hair follicle stem cell quiescence $(37)$, neuronal activity $(38,39)$, and glucose homeostasis (40). FOXP1 haploinsufficiency in humans leads to deformity in craniofacial structure and speech ability (41). In this study, we observed that Foxp1 deficiency in MSCs resulted in prototypical premature bone aging. These and additional results herein suggest that age-dependent bone loss may, in part, be orchestrated by the multifaceted action of FOXP1 during differentiation and senescence of MSCs.

\section{Results}

Foxp1 expression in BM MSCs declines with age. Nestin ${ }^{+}$MSCs in BM represent a population that constitutes an essential hematopoietic stem cell (HSC) niche (5). Immunohistochemical (IHC) examination revealed colocalization of nestin and FOXP1 in a subset of stromal cells within neonatal BM in vivo (Figure 1A). Colocalization was validated in vitro in a subpopulation of MSC in cultures (Supplemental Figure 1A; supplemental material available online with this article; https://doi.org/10.1172/JCI89511DS1). Of the 4 alternatively spliced FOXP1 isoforms in mouse tissues (42), we detected 3 in BM MSCs: FOXP1A, FOXP1D, and FOX- 
A

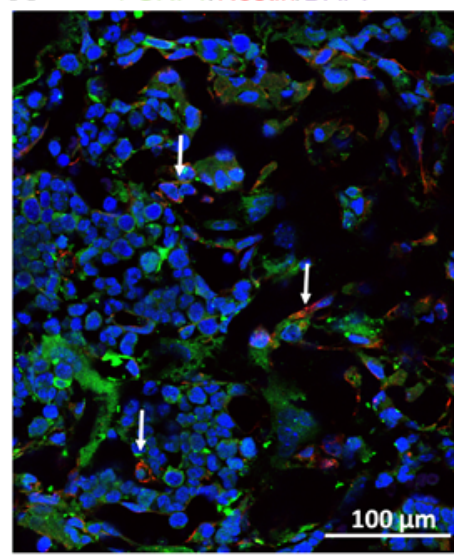

D

FOXP1

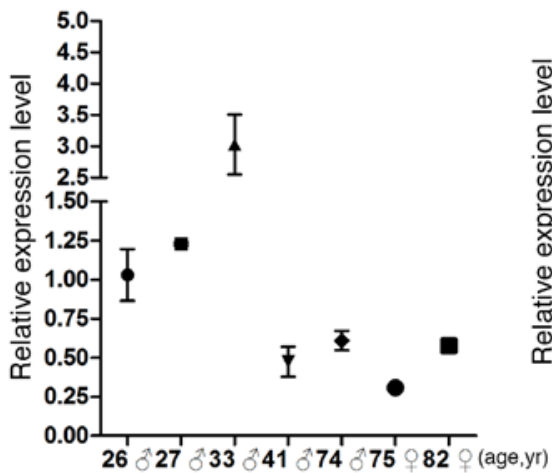

E
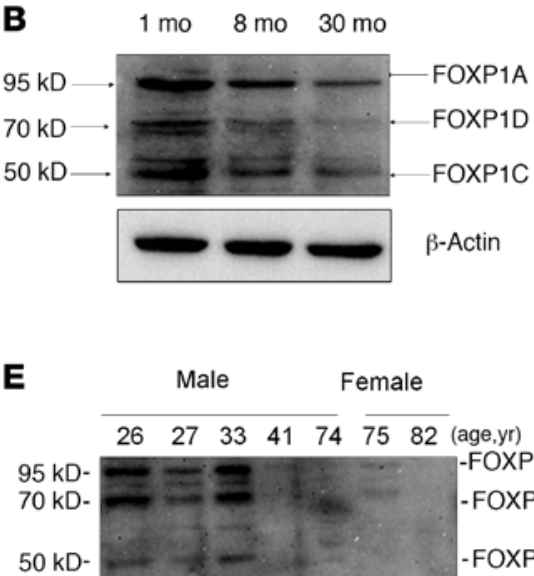

$50 \mathrm{kD}$

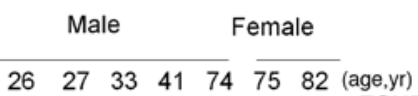

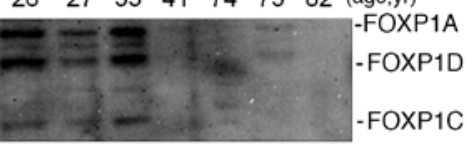

$\beta$-Actin
$\mathbf{F}$

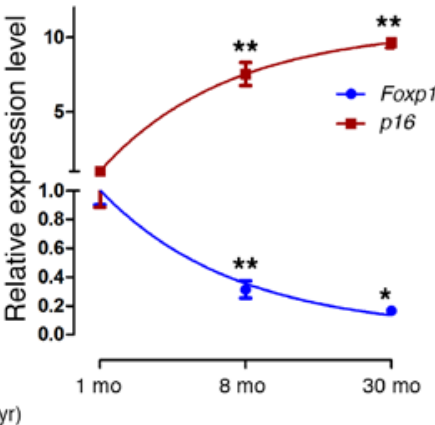

C Foxp1 transcription variants

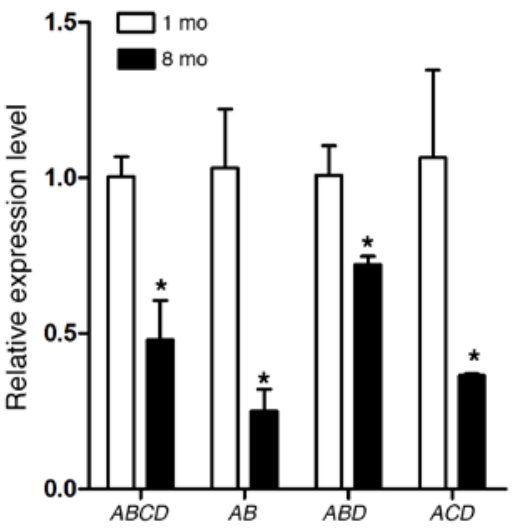

G

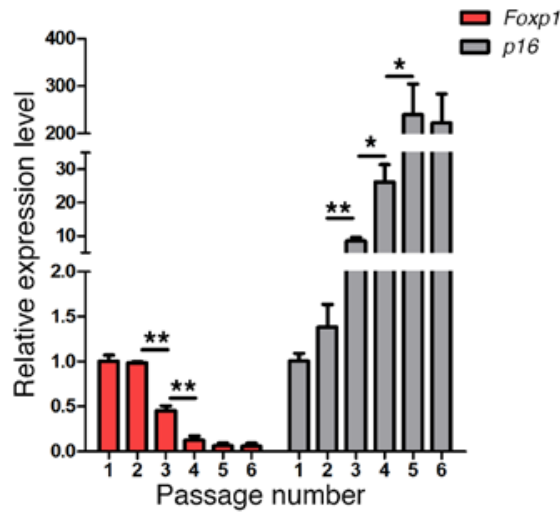

H mFoxp1 promoter

$-196$

1

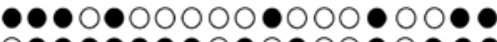

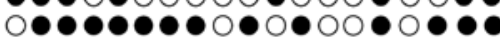

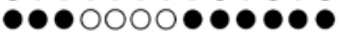

000000000000000000

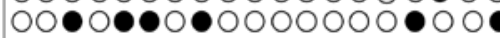

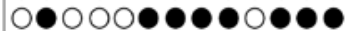

$1 \mathrm{mo}$

Methylation: $29 \%$

$12 \mathrm{mo}$

Methylation: $58 \%$

Figure 1. Foxp1 expression declines with age in BM MSCs. (A) Representative image of IHC analysis. IHC showed overlapping of FOXP1 (green) and nestin (red) in fibroblast-like cells adjacent to endosteal (white arrows) in neonatal BM. Scale bar: $100 \mu \mathrm{m}$. (B) Western blotting for the FOXP1 protein levels in BM MSCs. Western blotting for BM MSCs at 1, 8, and 30 months detected 3 major isoforms: FOXP1A (95 kD), FOXP1D (70 kD), and FOXP1C (50 kD). $n=3$. (C) qPCR for the relative expression of Foxp1 isoforms $A B, A B D, A C D$, and $A B C D$ in MPCs from BM of 1 and 8 months old. $n=3$. (D) Relative expression levels of FOXP1 in young and aged hMPC as detected by qPCR. (E) Western blotting for FOXP1 protein levels in human BM MSCs from donors ages 26, 27, 33, 41, 74, 75 and 82 years. (F) Inverse expressions of Foxp1 and p16 INK4A (p16) were assessed by qPCR in primitive MSCs obtained from BM of 1-, 8-, and 30-month-old mice. (G) Inverse correlation of Foxp1 and p16 IIK4A expression levels during in vitro expansion and passaging (P1-P6) of murine MSCs. $n=3$. (H) Methylation of CpG islands within the Foxp1 promoter variant 3 (-196 to 1) as detected by bisulfite sequencing in 1- and 12-month-old MSCs. Black circles represent methylated $\mathrm{CpG}$ islands and white circles unmethylated $\mathrm{CpG}$ islands. $n=3$. ${ }^{*} P<0.05 ;{ }^{*} P<0.01$.

P1C (Figure 1B and Supplemental Figure 1B). Foxp1 expression declined progressively in mice during their progression from adolescence (1 month) to aged ( 8 to 30 months; Figure 1B). To avoid the heterogeneity of MSCs in cultures, we validated decreased expression of FOXP1 in 1- and 18-month-old mouse BM sorted by flow cytometry as $\mathrm{CD} 29^{+} \mathrm{Sca} 1^{+} \mathrm{CD} 31^{-} \mathrm{CD} 45^{-}$Ter119- (Supplemental Figure 1, C and D). The expression decline was observed for all 3 FOXP1 isoforms (Figure 1C). Of note, FOXP1 expression in MSCs from human donors in ages ranging from 26 to 82 years was consistent with Foxp1 expression levels in mice (Figure 1, D and E). Curiously, Foxp1 levels in MSCs of different ages inversely correlated with those of the cellular senescence marker $p 16^{I N K 4 A}$ (Figure 1F). This inverse correlation was recapitulated in cultures of MSCs expanded in vitro for 6 passages (P1 to P6; Figure 1G). 
A
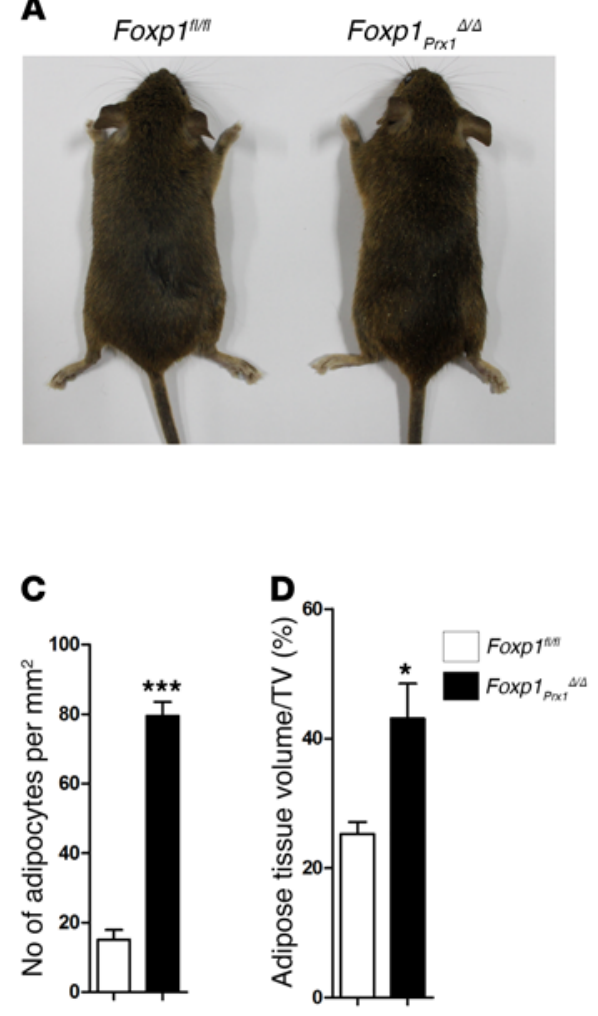

B

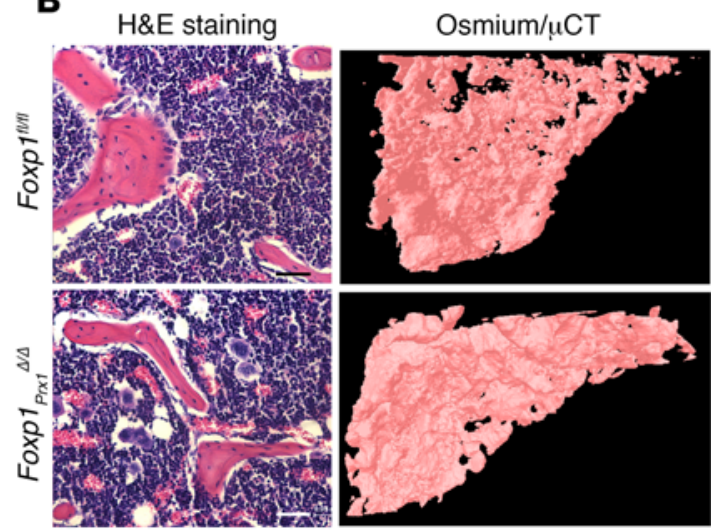

E

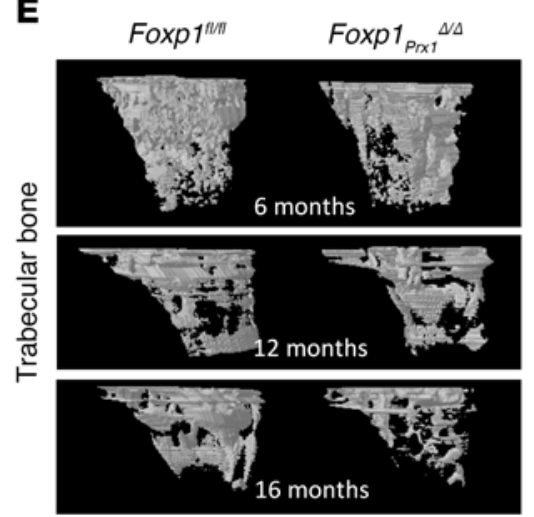

Figure 2. Foxp1 in MSCs regulates bone mass accrual in an age-dependent manner. (A) Representative images of Foxp $1_{P \times x}{ }^{4 / 4}$ mutant and Foxp $7^{f / / f l}$ mice at 3 months. (B) Left panel, representative H\&E staining for adipose tissue (yellow arrowheads) in trabecular bones of tibia at 8 months of age. Right panel, representative images of osmium tetraoxide staining followed by $\mu \mathrm{CT}$ analyses in tibia BM at 6 months. Scale bar: 50 $\mu \mathrm{m}$. (C and $\mathbf{D})$ Quantification of adipose droplet (C) and adipose tissue volume (D) in Foxp $1_{P r x 1} \Delta / \Delta B M$ as compared with WT controls. TV, total tissue volume. $n=5$. (E and $\mathbf{F})$ Representative images of trabecular (E) and cortical bones (F) of tibia by $\mu \mathrm{CT}$ analyses of $F o x p 1_{P r x /}{ }^{1 / 4}$ mutant and Foxp ${ }^{f / / f l}$ controls at 6, 12, and 16 months. (C) Representative $H \& E$ staining for primary ossification center of tibia bones at 6 months old. (H) Quantification of osteoblasts per trabecular bone surface in $\mathbf{C} . n=4$. Scale bar: $100 \mu \mathrm{m}^{*} P<0.05 ;{ }^{* *} P<0.01$; ${ }^{* * *} P<0.001$.

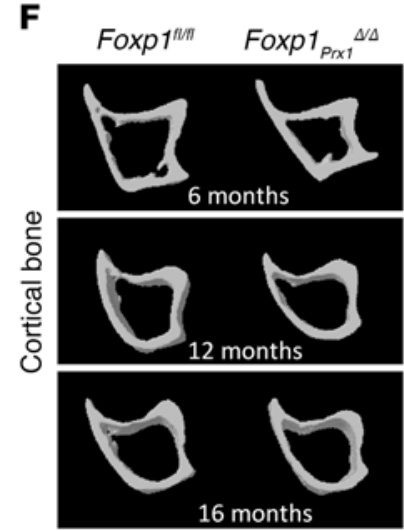

G

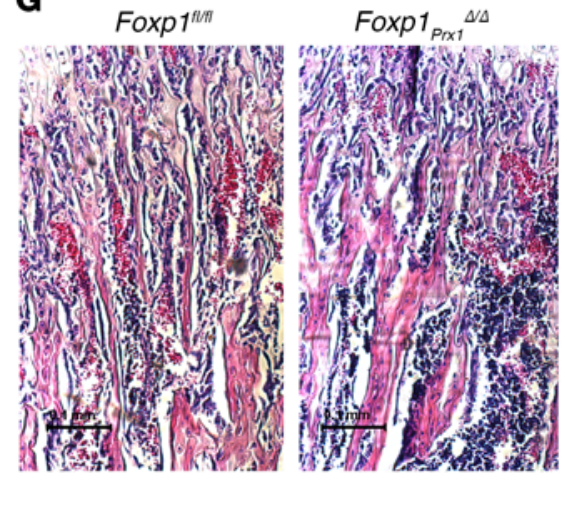

$\mathbf{H}$

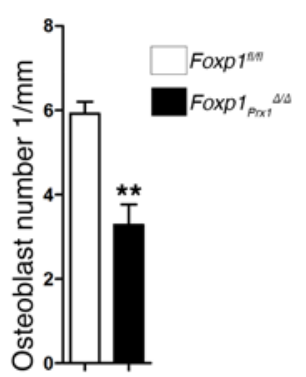

Meanwhile, the frequency of DNA methylation of CpG islands (43) within the proximal Foxp1 promoter (nucleotides -196 to +1 ) nearly doubled ( $29 \%$ to $58 \%$ ) as MSCs aged from 1 to 12 months (Figure 1H). These observations indicated that FOXP1 expression inversely correlates with the progression of MSC aging.

Ablation of Foxp1 in MSCs results in age-dependent bone loss. To test the role of FOXP1 in MSC differentiation and senescence, we inactivated it specifically in early BM MSCs by crossing a floxed ( $f l)$ allele Foxp1 $1^{f l f l}$ (31) with Prx1-Cre (44). Quantitative PCR (qPCR) and Western blotting confirmed efficient depletion of Foxp1 expression (Supplemental Figure 2, A and B). Prx1-Cre Foxp1 $1^{f / f l}$ conditional KO mice (hereafter designated as Foxp $1_{P r x l} \Delta / \Delta$ ) showed no changes in size, weight, or growth as compared with Foxp $1^{f / f l}$ controls (Figure 2A and data not shown). Notably, H\&E staining revealed relative enrichment of adipose tissues within the BM of
Foxp $1_{P r x 1}{ }^{4 / \Delta}$ mutants (Figure 2, B and C). In addition, the relative volume of BM adipose tissue was increased in Foxp $1_{P r x 1}{ }^{4 / \Delta}$ mutants, as revealed by microcomputer tomography $(\mu \mathrm{CT})$ analyses following osmium staining (Figure 2, B and D). $\mu \mathrm{CT}$ analyses further showed that Foxp $1_{P r x 1}{ }^{\Delta / \Delta}$ mice displayed age-related bone loss as compared with Foxp1 $1^{f / f l}$ controls at 6, 12, and 16 months (Figure 2, $\mathrm{E}$ and $\mathrm{F}$ ), as evidenced by a gradual decrease in bone mineral density (BMD) and in trabecular bone numbers (Supplemental Figure 2, $\mathrm{C}$ and $\mathrm{D}$ ). This impaired bone mass accrual also was detected in 6-month-old female Foxp $1_{P r x 1}{ }^{4 / \Delta}$ mutants (Supplemental Figure $2 \mathrm{E}$ ). Histomorphometric analysis showed that osteoblasts per $\mathrm{mm}$ trabecular bone surface were decreased in mutants (Figure 2, G and $\mathrm{H}$ ). The decline in BMD did not arise from enhanced osteoclast activity, as evidenced by tartrate-resistant acid phosphatase (TRAP) $(n=3)$ staining of osteoclasts in trabecular bones and 
A

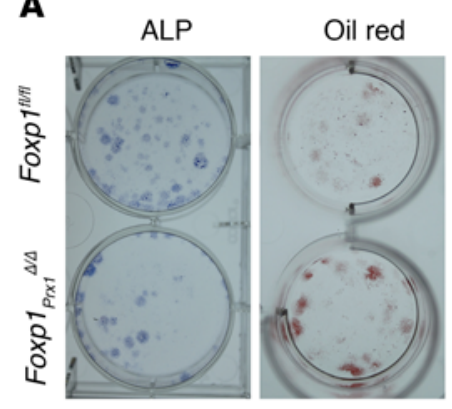

B

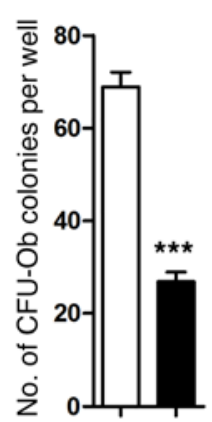

$\mathbf{F}$

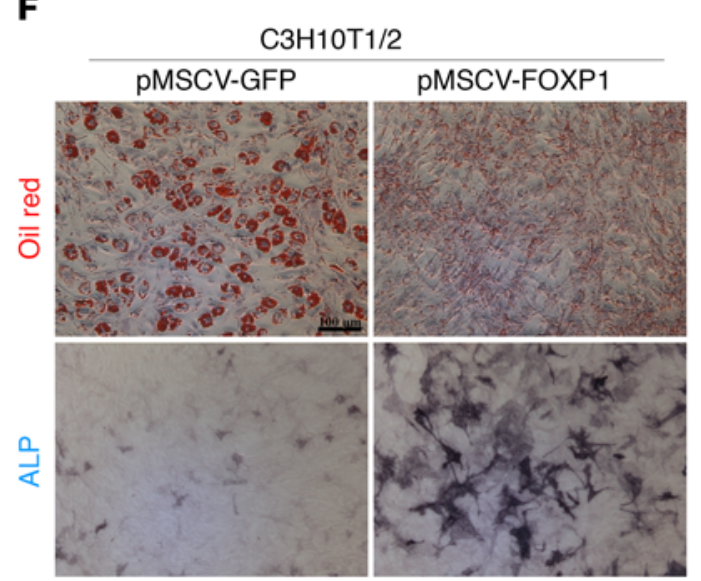

C

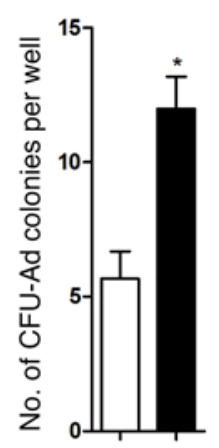

D

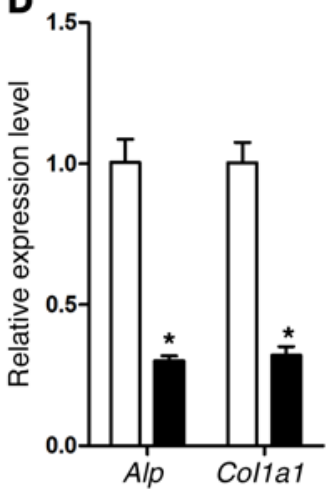

E

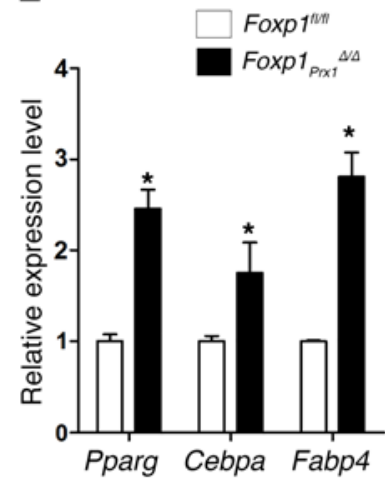

G

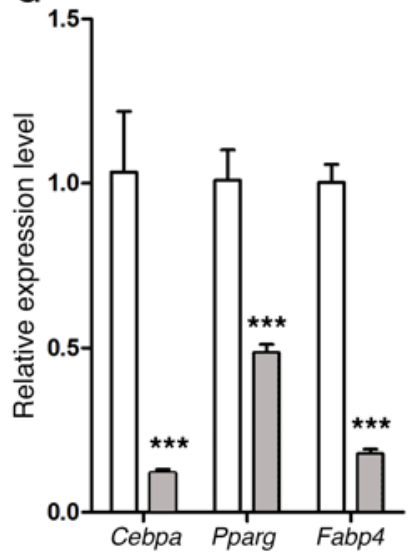

H

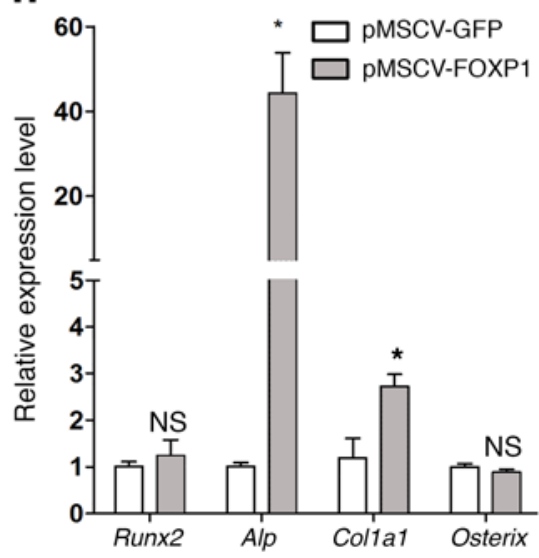

Figure 3. FOXP1 modulates cell-fate choice of MSCs between adipocytes and osteoblasts. (A) The osteogenic and adipogenic potential of MSCs from FOXP1 mutant BM was assessed 14 days after induction of differentiation by ALP and oil red 0 staining. $n=5$. (B and $\mathbf{C}$ ) Quantification of the frequencies of osteoblast clones (CFU-Ob) and adipocyte clones (CFU-Ad) following induction in A. $n=3$. (D and E) Expression of bone (Alp, Col1a1) and fat (Pparg, Cebpa, and Fabp4) markers as assessed by quantitative reverse-transcriptase PCR (qRT-PCR) of uninduced MSCs in BM. $n=3$. (F) Foxp1 was overexpressed in C3H10T1/2 cells by retrovirus (pMSCV-FOXP1) and cultured in differentiation medium. Cell differentiation was assessed 6 days after adipogenic induction by oil red 0 staining or 14 days after osteogenic induction by ALP staining. $n=3$. Scale bar: $100 \mu \mathrm{m}$ (G) The expression of adipogenic markers (Cebpa, Pparg, and Fabp4) was analyzed 6 days after adipogenic induction of FOXP1-expressing C3H10T1/2 cells. $n=3$. (H) The expression of bone markers (Runx2, Alp, Col1a1, and Osterix) was assessed by qPCR 14 days after osteogenic culture of FOXP1-overexpressing C3H10T1/2 cells. $n=3$. ${ }^{*} P<0.05 ;{ }^{* *} P<0.001$.

FACS analysis of osteoclast progenitor cells in BM at 3 months (Supplemental Figure 3). Collectively, these perturbations in bone and adipose tissue indicated that Foxp1-deficient bones displayed premature aging-related characteristics.

Foxp1 regulates the potential of MSCs to differentiate into adipocytes and osteoblasts. To investigate the role of Foxp1 in cellfate choice, MSCs were flushed from BM and induced to differentiate in vitro (detailed in Methods). Within 14 days following adipogenic or osteogenic CFU fibroblast (CFU-F) induction, the number of Foxp $1_{P r x l} \Delta / \Delta$ adipocyte CFUs (Ad-CFUs) significantly increased, whereas the number of mutant osteoblast CFUs (Ob-CFUs) decreased relative to controls (Figure 3, A-C). This fate switch in MSC differentiation potential was validated by parallel downregulation of osteoblast-specific transcripts ( $\mathrm{Alp}$, Col1a1) and upregulation of adipocyte-related markers (Pparg, Cebpa, and Fabp4) (Figure 3, D and E).

Next, we stably overexpressed Foxp1 in C3H1OT1/2 mesenchymal cells by retroviral transduction. We then assessed cell differentiation either by oil red $O$ staining 6 days after adipogenic induction or by alkaline phosphatase (ALP) staining 14 days after osteogenic induction (Figure 3F). As predicted by our KO results, enforced expression of Foxp1 significantly enhanced osteogenic differentiation at the expense of adipogenic differentiation, as evidenced by increased or decreased levels, respectively, of the aforementioned osteoblast and adipocyte marker transcripts (Figure 3, G and H). Next, we transfected primary MSCs with a phosphorylated murine stem cell virus-driven (pMSCV-driven) FOXP1 retrovirus and examined differentiation by staining and expression of the same molecular markers employed for Figure 3, $\mathrm{G}$ and H (Supplemental Figure 4). Again, we observed that FOXP1 potentiated the osteogenic differentiation of MSCs.

To further validate the action of Foxp1 in MSC differentiation, we generated Nestin-Cre Foxp $1^{f / f l}$ conditional KO mice (designated as Foxp $1_{\text {Nes }}^{4 / \Delta}$ ). Nestin-Cre targets a distinct population of MSCs within BM that constitute niches for HSC (5). Foxp $1_{\text {Nes }}{ }^{4 / \Delta}$ mutant mice displayed far more severe defects in bone growth than did Foxp $1_{P r x 1}{ }^{4 / \Delta}$ mice (Supplemental Figure 5A and Supplemental Figure 6A). Foxp $1_{\text {Nes }}{ }^{4 / \Delta}$ mutants died within 6 weeks and displayed obvious growth arrest relative to controls, perhaps as a result of defects in neuronal activity $(38,39)$. In Foxp $1_{\text {Nes }}{ }^{4 / \Delta}$ mutants, the 
osteogenic and adipogenic potential of MSCs was similarly altered, as evidenced by impaired bone formation (Supplemental Figure 5, $\mathrm{B}$ and C), enrichment of adipose tissue (Supplemental Figure 5, C and D), and altered numbers of Ad-CFU and Ob-CFU following MSC differentiation (Supplemental Figure 6, D-F) as well as by increased expression levels of PPAR $\gamma$ and its downstream target gene, Fabp4 (45) (Supplemental Figure 6G).

Given that Prx1-Cre and nestin-Cre target both osteoblast and osteocytes, the defects in bone mass accrual in Foxp $1_{P r x 1}^{4 / \Delta}$ and Foxp $1_{\text {Nes }}{ }^{\Delta / \Delta}$ mutants may result from loss of function of either. Thus, we crossed Foxp1 $1^{f l f l}$ mice with Col2a1-Cre (46), which targets osteoprogenitors and osteoblasts. We detected no changes in bone mass accrual as measured by $\mu \mathrm{CT}$ analyses in Foxp $1_{\text {Colzal }}{ }^{4 / 4}$ mutants as compared with WT controls at 3 months of age (Supplemental Figure 7, A and B). In addition, the loss of FOXP1 did not influence growth plate organization or chondrogenic differentiation during MSC induction (Supplemental Figure 7, C and D). These observations indicate that FOXP1 primarily exerts its influence on MSCs rather than chondrocytes or osteoblasts. Taken together, the defects in Foxp $1_{P r x 1}{ }^{4 / \Delta}$ and Foxp $1_{\text {Nes }}{ }^{4 / \Delta}$ mutant mice indicate that Foxp1 deficiency in MSCs favors adipogenesis over osteogenesis. Consequentially, FOXP1 is essential in establishing fate choice between bone and adipose tissue in vivo.

Foxp1 controls adipocyte and osteoblast differentiation by interacting with $C E B P \beta / \delta$ and RBPjк. Adipogenic differentiation of MSCs is initiated by a program of sequential activation of the transcriptional factors $\mathrm{CEBP} \beta / \delta, \mathrm{CEBP} \alpha$, and $\operatorname{PPAR} \gamma(10)$. We observed via coimmunoprecipitation (co-IP) assays that FOXP1 interacts with CEBP $\beta$ and CEBP $\delta$ in 3T3-L1 cells (Figure 4, A and B) and in BM MSCs (Figure $4 \mathrm{C}$ ). FOXP1 and CEBP $\beta / \delta$ colocalize within nuclei following transfection of $\mathrm{C} 3 \mathrm{H} 10 \mathrm{~T} 1 / 2$ cells with vectors encoding either FOXP1-His/CEBP $\beta / \delta$ or FOXP1/CEBP $\beta / \delta$-Flag (Figure 4D and Supplemental Figure $8 \mathrm{~B}$ ).

$\mathrm{CEBP} \alpha$ and $\mathrm{CEBP} \beta / \delta$ act upstream to induce Pparg transcription during terminal adipocyte differentiation (47). Luciferase reporter assays in $\mathrm{C} 3 \mathrm{H} 10 \mathrm{~T} 1 / 2$ cells revealed that transactivation of Pparg-Luc by either CEBP $\alpha$ or $\mathrm{CEBP} \beta / \delta$ was repressed by FOXP1 (Figure 4E and Supplemental Figure 8A). In contrast, expression of PPAR $\gamma$ and FABP4 was relatively elevated in Foxp1-deficient MSCs compared with controls (Figure 4, F and G). We identified a consensus FOXP1-binding site -1112 bp upstream of the Pparg transcriptional start site (data not shown). ChIP-PCR indicated strong FOXP1 binding to that site (Figure 4, $\mathrm{H}$ and I), indicative of direct FOXP1 repression of Pparg transcription. Consistent with this interpretation, overexpression of FOXP1 repressed Pparg and Fabp 4 transcription following adipogenic differentiation of C3H1OT1/2 cells (Figure 3G). Collectively, our observations suggest that a FOXP1-CEBP $\beta / \delta$ complex attenuates Pparg transcription to restrain adipogenic differentiation of MSCs.

Notch/recombination signal binding protein for immunoglobulin $\kappa \mathrm{J}$ region (RBPj $)$ signaling maintains MSC identity by suppressing osteoblast differentiation $(48,49)$. Via co-IP, we detected interaction of FOXP1 with RBPjк in both C3H10T1/2 mesenchymal cells and BM MSCs (Figure 4, J and K). Colocalization of FOXP1 and RBPjк protein within C3H1OT1/2 nuclei was also detected (Figure 4D and Supplemental Figure 8B). Next, we observed that, through this interaction, FOXP1 repressed the acti- vation of RBPJk-Luc via the intracellular domain of Notch (NICD) (Figure 4L). Additionally, Foxp $1_{P r x 1}{ }^{4 / \Delta}$ BM MSCs exhibited elevated expression of Hey1 and Heyl -2 quintessential downstream targets of Notch signaling (Figure 4M). Accordingly, enforced expression of FOXP1 in C3H10T1/2 cells repressed expression of Heyl and Heyl (Figure 4N). These findings suggest that FOXP1 promotes osteogenic differentiation of MSCs through repression of Notch signaling during postnatal skeletal aging. However, further studies are needed to more robustly confirm this hypothesis.

Foxp1 attenuates MSC senescence through repression of $p 16^{\text {INK } 4 A}$ transcription. In terms of stem cell replication capacity, explants of Foxp $1_{\text {Prxl }}{ }^{4 / \Delta}$ MSCs displayed marked arrest in population doubling measured in vitro at 1 and 6 months (Figure 5A). Following 48-hour BrdU pulse-chase, FACS analyses revealed a smaller proportion of $\mathrm{BrdU}^{+} \mathrm{MSCs}\left(\mathrm{CD} 31^{-} \mathrm{CD} 45^{-} \mathrm{CD} 29^{+} \mathrm{Sca}^{+}\right)$in the BM of Foxp $1_{\text {Prxl }}{ }^{4 / \Delta}$ mice (Figure 5, B and C). We further observed that expression levels of cell cycle inhibitors $p 16^{I N K 4 A}, p 21$, and p27 were relatively increased in MSCs following isolation from Foxp1-deficient BM (Figure 5D). However, the number of CFU-F colonies began to progressively decrease at 6 months (Figure 5, $\mathrm{E}$ and $\mathrm{F}$ ), consistent with a reduction in self-renewal ability upon loss of Foxp1. Expression levels of p16 ${ }^{\text {INK4A }}$, a marker for cellular senescence (18), were also significantly increased (Figure 5G). Yet expression of another 2 aging-associated markers, H3K9me3 and LAP2 $\beta$ (50), was decreased in Foxp1-deficient MSCs (Figure $5 \mathrm{G})$. Whereas second-passage MSC cultures of Foxp $1_{P r x 1}{ }^{4 / \Delta} \mathrm{BM}$ had relatively fewer $\mathrm{Ki}^{-} 7^{+}$cells than Foxp $1^{f / f l}$ controls (Figure 5, $\mathrm{H}$ and I), a greater number of $\gamma \mathrm{H} 2 \mathrm{AX}^{+}$and senescence-associated $\beta$-galactosidase activity $\left(\mathrm{SA}-\beta\right.$-gal ${ }^{+}$) cells as well as increased ROS levels were detected in mutant MSC cultures (Figure 5, H-K, and Supplemental Figure 8D). This decreased distribution of nuclear LAP2 $\beta$ in Foxp1-deficient MSCs was confirmed by high-resolution IHC (Figure $5 \mathrm{H}$ ) and was consistent with the Western blot data (Figure 5G). These in vivo and in vitro experiments indicated that Foxp1-deficient MSCs undergo a decline in self-renewal capacity. This, in turn, leads to swift accumulation of DNA damage and premature senescence during expansion.

Tight transcriptional control of the $p 16^{\text {INK4A }}$ locus is important in regulating senescence of a number of adult stem cell lineages during aging $(13,14,51,52)$. Promoter occupancy analysis identified FOXP1-binding sites within the $p 16^{\text {INK4A }}$ promoter (-1701 to-1695, Figure 6A). Luciferase reporter assays employing p16-Luc (driven by its essential $2.8 \mathrm{~kb}$ promoter) revealed that FOXP1 repressed activity of the $p 16^{I N K 4 A} \mathrm{WT}$, but not the FOXP1-binding site-mutated $p 16^{\text {INK4A }}$ reporter (Figure 6, B and C). These findings suggested that FOXP1 restrains MSC senescence through repression of $p 16^{\text {INK4A }}$ transcription during skeletal aging.

To further test this hypothesis, we investigated the phenotypes of doublydeleted Foxp1 and $p 16^{I N K 4 A}$ conditional KO mice (Foxp $1_{P r x 1}{ }^{4 / 4}$ $\left.p 16^{-/}\right)$. Even though singular loss of $p 16^{I N K 4 A}$ had shown no effect on MSC expansion or bone growth at 3 months of age as described previously (52), we observed a partial rescue of the replicative capacity in Foxp $1_{P r x 1}{ }^{4 / \Delta} p 16^{-/-}$double-mutant mice (Figure 6D). $\mu \mathrm{CT}$ analyses of bone parameters showed that, as compared with single Foxp $1_{P r x 1}{ }^{4 / \Delta}$ mutants, double-mutant mice displayed significantly increased volume, number, and BMD of trabecular bones (Figure 6, E and F). We also observed decreased anatomical separation of trabecular, 
A

FOXP1-His CEBP $\beta$-Flag

IP: His

IP: Flag

Input

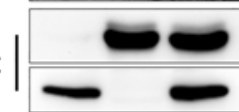

IB: Flag

IB: His

IB: Flag IB: His

B

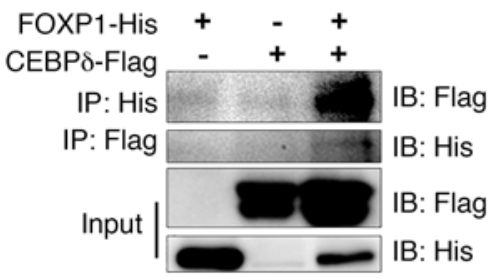

C

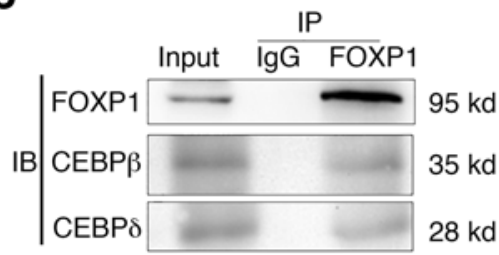

H

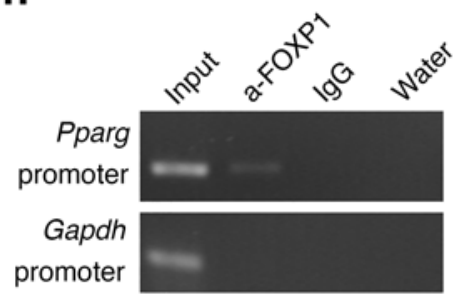

D
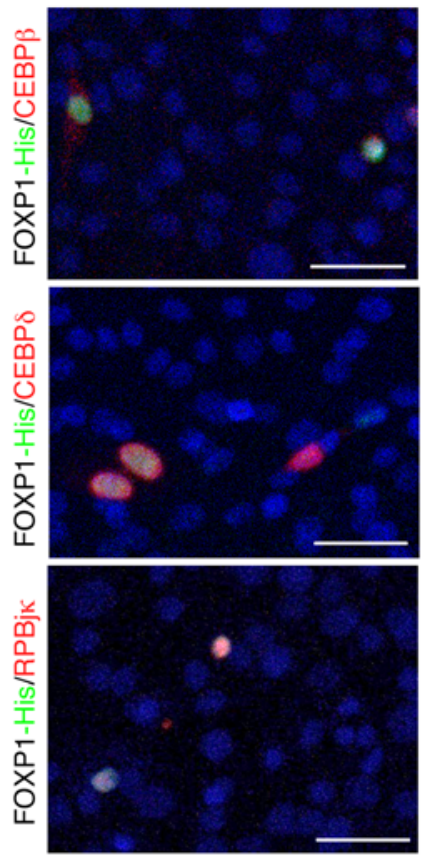

$28 \mathrm{kd}$

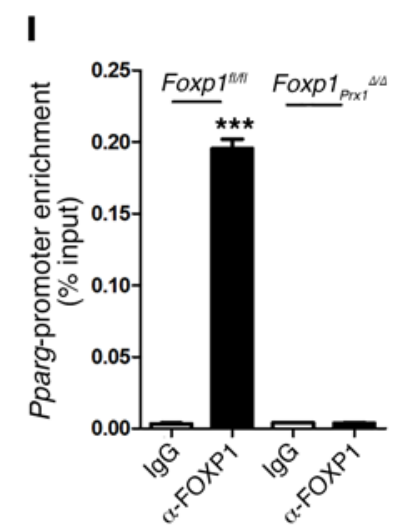

J

FOXP1-His + - +

RBPjк-His - + +

IP: His IB: Flag

IP: Flag $\quad= \pm$ IB: His

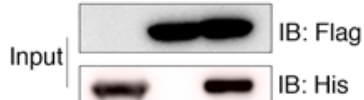

$\mathbf{K}$

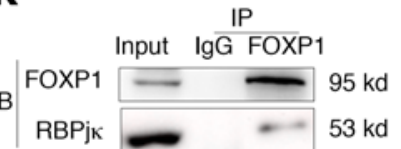

E

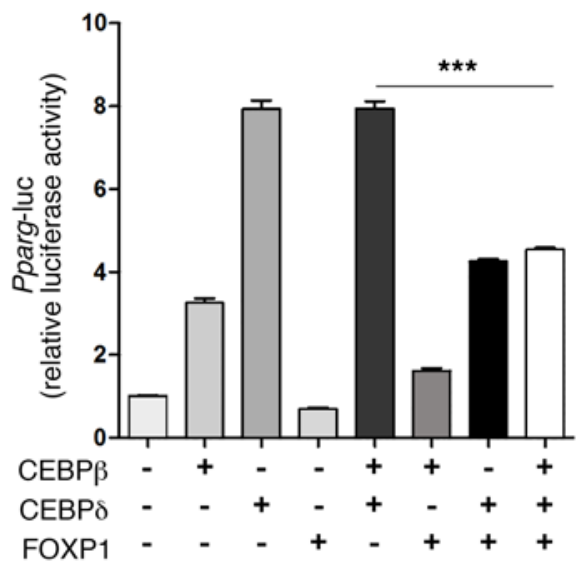

$\mathbf{F}$

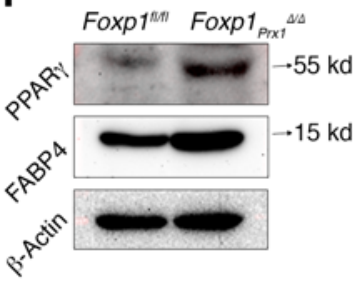

G

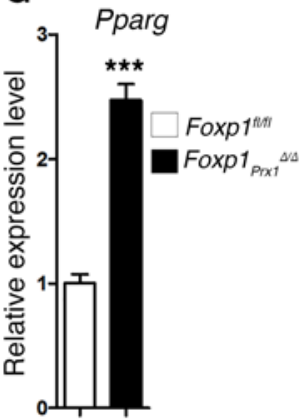

$\mathbf{L}$

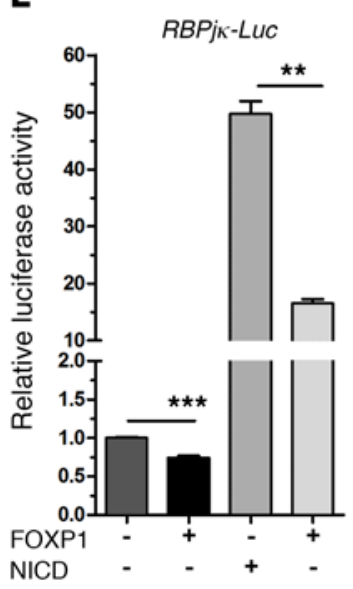

M

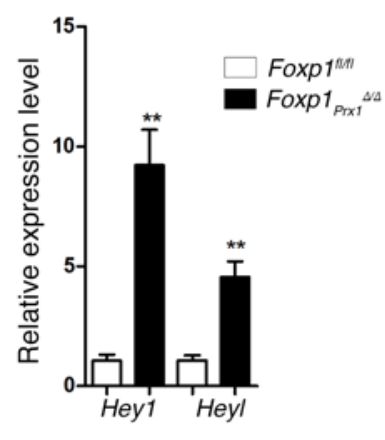

N

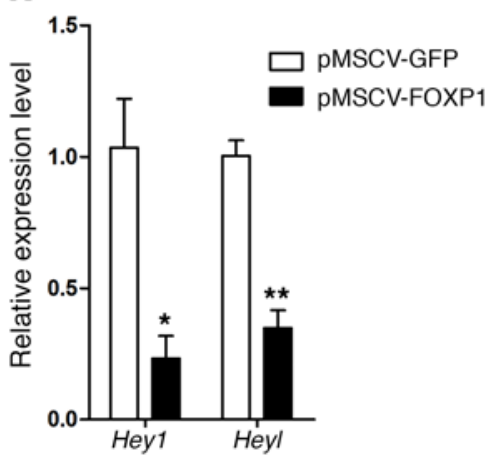


Figure 4. FOXP1 controls adipocyte and osteoblast differentiation by interacting with CEBP $\beta / \delta$ and RBPjк. (A and B) In vitro co-IP of FOXP1-His with CEBP $\beta$-Flag (A) or CEBP $\delta$-Flag (B) following transfection into 3T3-L1 cells. (C) Western blotting detection of CEBP $\beta$ and CEBP $\delta$ in FOXP1 immunoprecipitates from primary BM MSCs. (D) Colocalization of FOXP1 and CEBP $\beta$, CEBP $\delta$ or RBPjא in the nuclei of C3H10T1/2 cells transfected with FOXP1-His

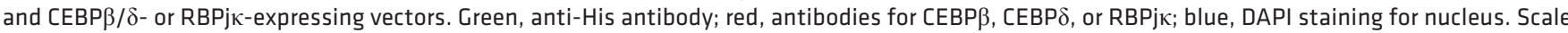
bar: $50 \mu \mathrm{m}$. (E) FOXP1 represses the transactivation ability of CEBP $\beta$ and CEBP $\delta$ in inducing Pparg-Luc luciferase activity in C3H10T1/2 cells. $n=3$. (F) Western blot detection of PPAR $\gamma$ and FABP4 levels in Foxp $1_{P r x x} / / 4$ mutant BM at 8 months. (C) qRT-PCR of Pparg mRNA levels in MSCs. $n=3$. (H and I) Promoter occupancy of Pparg gene as assessed by anti-FOXP1 ChIP-PCR in MSCs. (J and K) Co-IP of FOXP1 with RBPjk in C3H10T1/2 cells (J) and BM MSCs (K). (L) FOXP1 repression of luciferase activity as judged by Rbpjk-Luc reporter activity following the induction by NICD in C3H10T1/2 cells. $n=3$. ( $\mathbf{M}$ and $\mathbf{N}$ ) qPCR confirms relatively higher expression levels of Hey1 and Heyl in MSCs from Foxp $1_{\text {Prx } 1 / 4}{ }^{4 / 4}$ tant BM (M) or C3H10T1/2 cells transfected with pMSCV-FOXP1 (N). $n=3 .{ }^{*} P<0.05 ;{ }^{* *} P<0.01 ;{ }^{* *} P<0.001$

but not of cortical, bones (data not shown). These results suggest that deletion of $p 16^{I N K 4 A}$ partially compensates for defects in the replicative function of Foxp1-deficient MSCs.

Overexpression of FOXP1 in human mesenchymal progenitors augments their replication capacity. Given that Foxp1 expression correlated with the aging progress of murine MSCs, we tested whether overexpression of FOXP1 could compromise senescence in human mesenchymal progenitor cells (hMPCs). Overexpression of lentiviral FOXP1 (Supplemental Figure 8E) within hMPCs collected from donors of both sexes at ages ranging from 27 to 82 years significantly augmented their replicative capacity, as assessed by measurement of population doubling (Figure 7, A-D). Of note, overexpression of FOXP1 hMPCs from a 74-year-old donor increased their expansion capacity to levels comparable to that of hMPCs from a 27-year-old donor (Figure 7D). This was coupled with a dramatic repression of $p 16^{1 N K 4 A}$ transcription within the fifth passage of FOXP1-overexpressing hMPCs (Figure 7E). We also observed that hMPCs transduced with Foxp1 had greater osteogenic potential and reduced adipogenic potential, as evidenced by ALP and oil red O staining, by qPCR for osteogenic markers (ALP, COL1A1, HEY1 and HEYL), and by Western blotting for adipogenic markers (PPAR $\gamma$ and FABP4) (Figure 7, F-H). Collectively, our results indicate that FOXP1 is capable of augmenting the replication capacity of hMPCs irrespective of their age.

\section{Discussion}

Studies of mesenchymal progenitor-associated transcription factors in humans first reported that FOXP1 expression was higher in hMPCs than in their differentiated progenies (53). Expanding upon that observation, we identified FOXP1 as a dose-dependent orchestrator of MSC senescence and differentiation potency during skeletal aging. Our data suggest a model in which high levels of FOXP1 expression in young MSCs prevent their premature senescence, leading to an outcome that favors bone formation over adipogenesis (Figure 7I). In aged MSCs, a decline in FOXP1 levels alleviates negative regulation of $p 16^{I N K 4 A}$ transcription and adipogenesis to promote the aging of MSCs (Figure 7I).

In a previous study, neither FOXP1 nor $p 16^{I N K A A}$ expression was found to significantly decrease in MSCs from middle-aged to elderly donors (54). Perhaps FOXP1 expression in MSCs from middle-aged donors had already begun to decrease when their measurements were made. In support of this interpretation, Foxp1 expression in 8 -month-old murine MSCs decreased approximately $60 \%$ as compared with that in 1-month-old mice (Figure 1, B and F).

Epigenetic modifications, such as promotor-associated DNA methylation, appear to be a hallmark of MSC senescence
(55). DNA methylation within the Foxp1 promoter region is increased with age, thereby potentially contributing to the progressive downregulation of Foxp1 expression. That FOXP1 directly represses Pparg transcription in MSCs may, at least in part, explain the chronic increase of PPAR $\gamma$ expression in senescent MSCs (56). PPAR $\gamma$ acts as a master regulator of adipocyte differentiation by suppressing osteoblast differentiation (57-59). Thus, we contend that a decrease in FOXP1 levels, coupled with augmentation of PPAR $\gamma$ expression, contributes to the increased adiposity in aged bones. We also found that FOXP1 may restrain the osteogenic potential of MSCs by suppressing Notch signaling (Figure 4, J-N). Collectively, our data indicate that FOXP1 acts as a critical effector of aging by controlling adipo-osteogenic balance during MSC differentiation.

A previous study claimed that siRNA-mediated knockdown (KD) of FOXP1 impaired adipogenesis, but not osteogenic differentiation, when tested in vitro (53). However, the efficacy and specificity of FOXP1 KD - a critical parameter for drawing this conclusion - were not presented in this study. Given the highly conserved sequence and redundant functions of FOXP1/2/4 isoforms (60), one cannot exclude the possibility that inefficient and/ or mistargeted KD led to this conclusion.

Our results, derived both from in vivo and in vitro analyses, demonstrated a critical function for FOXP1 in fate choice of MSC differentiation. In addition, elevation of FOXP1 expression in aged hMPCs reversed their decline in expansion capacity and osteogenic potential. The current anabolic targets for osteoporosis, such as parathyroid hormone (PTH) or Wnt agonists, mainly promote osteoblastogenesis in bone formation (61). The multiple actions of FOXP1 in regulating MSC plasticity and senescence engender it as a potential anabolic target for osteoporosis therapy.

A central finding of our study is that FOXP1 attenuates MSC aging by directly regulating $p 16^{I N K A A}$ transcription. Most relevant to this, FOXP1 overexpression has been associated with a variety of cancers (62-71). Given that the state of senescence can protect cells against the development of cancer (14), we submit that our findings may be instructive in understanding FOXP1-associated mechanisms of multiple tumorigenesis. Further studies are needed to address how the FOXP1/p16 cascade protects multiple cell lineages against premature senescence or unlimited overgrowth.

\section{Methods}

Mice. Foxp1 $1^{f / f l}$ (29), $p 16^{-/-}$(17), Prx1-Cre (72), and nestin-Cre (5) mice were generated as described elsewhere. The genetic backgrounds of all KO mice were uniform mixtures of 129S1/SvIMJ and C57BL/6. Mice were bred in pathogen-free conditions. 

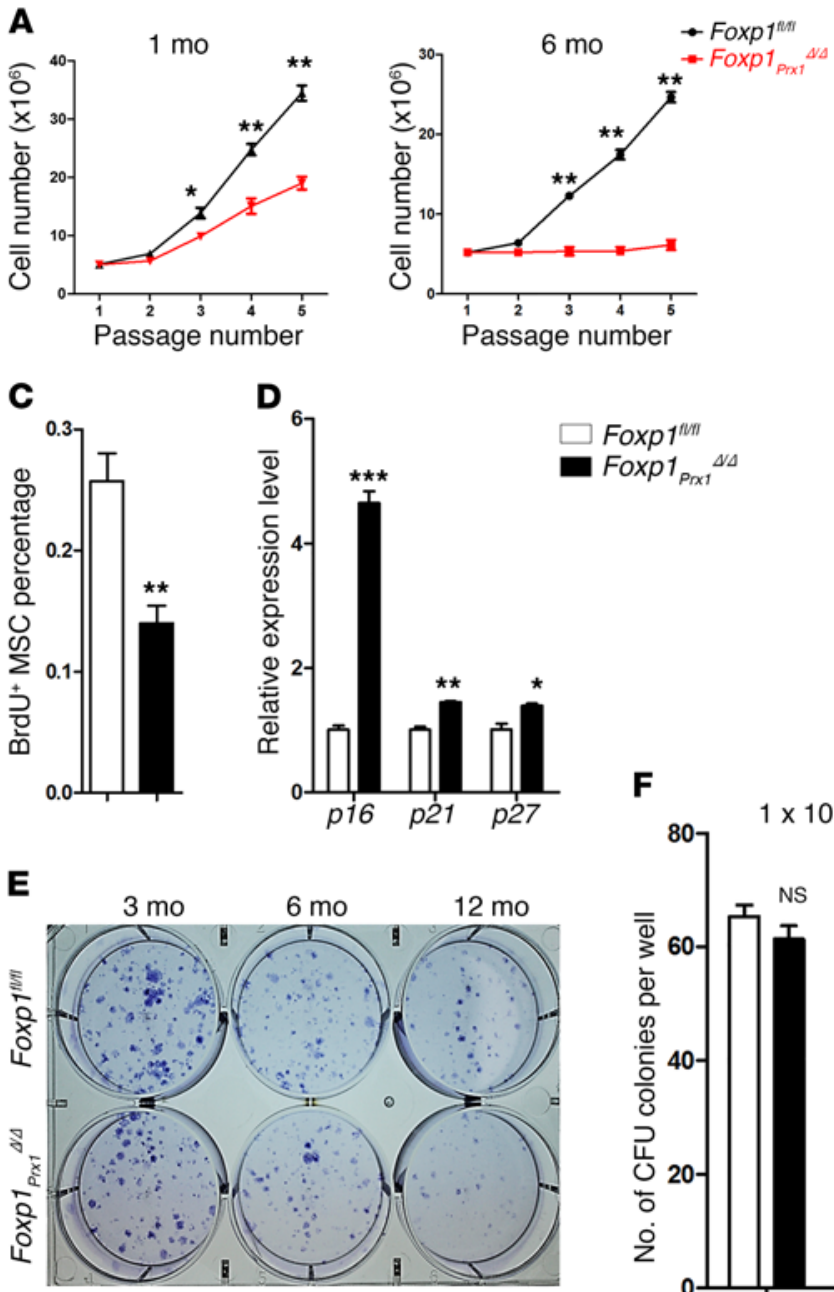

F
B

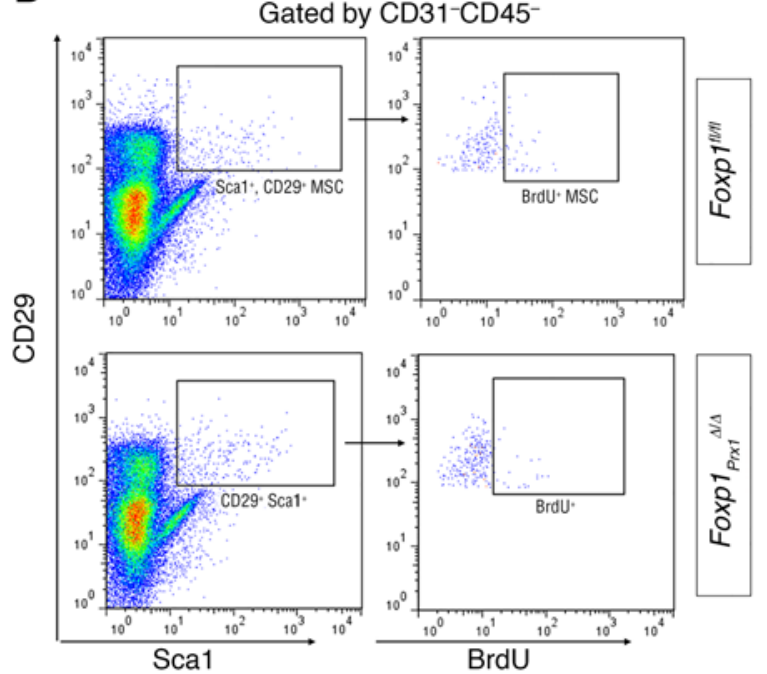

G

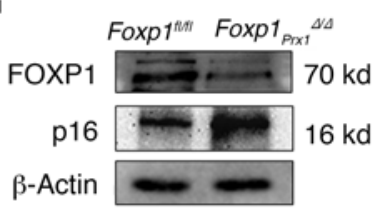

$1 \times 10^{6}$ cells per well

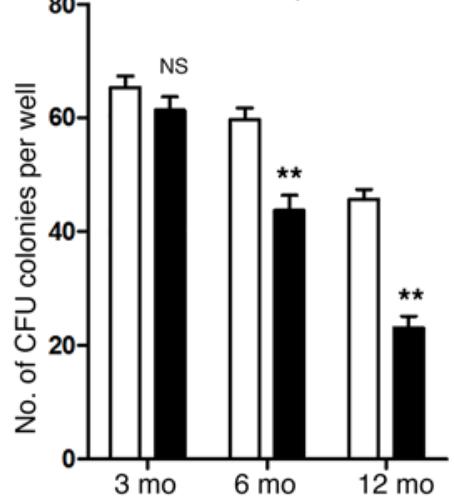

Foxp $1^{\text {thr }}$

Foxp1 ${ }_{P|x|}^{\text {AS }}$

Foxp1 $1^{\text {InI }}$ Foxp1 $1_{\text {Prx } 1}{ }^{\Delta / 4}$

H3K9me3

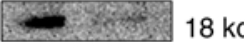

LAP2 $\beta$

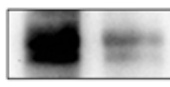

$55 \mathrm{kd}$

$\beta$-Actin

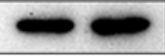

H
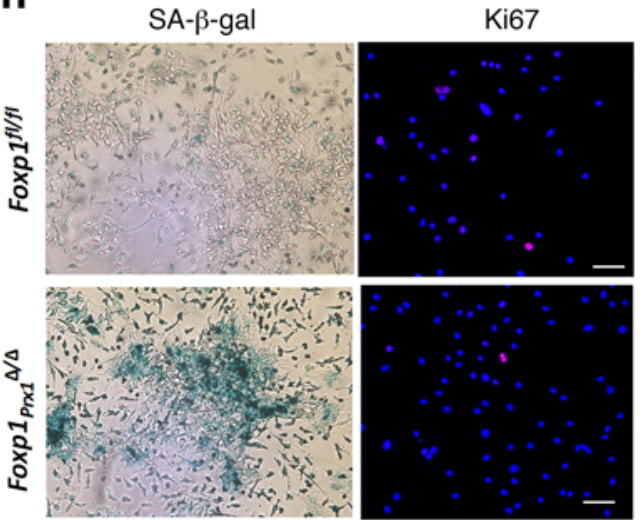
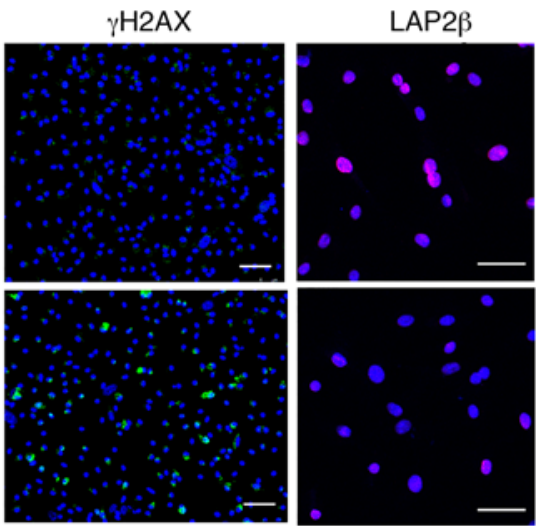

Figure 5. Ablation of Foxp1 results in premature senescence of MSCs. (A) In vitro population doublings of MSCs from Foxp1 ${ }_{P \times x}{ }^{4 / 4}$ BM at 1 and 6 months. $n=4$. (B) FACS analysis dot plot of BrdU+ labeled MSCs (defined as CD31-CD45-CD29+Sca1+) following 48-hour pulse-chase in BM of 3-month-old mice. $n=3$. (C) Quantification of the percentages of BrdU+ MSCs in 3-month-old mice. $n=4$. (D) qPCR assessment of cell-cycle inhibitor (p16 IIK4A,$p 21$, and p27) expression in BM MSCs. $n=3$. (E) Giemsa staining for the CFU-F colonies of BM MSCs from Foxp $1_{P r x]}{ }^{4 / 4}$ mutant mice at 3, 6, and 12 months. $n=3$. (F) Quantification of the number of CFU-F colonies in $\mathbf{E}$. $n=3$. (G) Western blot detection of FOXP1, p16 ${ }^{1 \mathrm{NK} 4 \mathrm{~A}}, \mathrm{H} 3 \mathrm{~K} 9 \mathrm{me}$, and LAP2 $\beta$ protein levels in BM MSCs. (H) SA- $\beta$-gal staining, Ki67, $\gamma \mathrm{H} 2 \mathrm{AX}$, and LAP2 $\beta$ immunostaining of the second passage of MSCs. Scale bars: $50 \mu \mathrm{m}$. (I and J) Quantification of Ki67 ${ }^{+}$and $\gamma \mathrm{H} 2 \mathrm{AX} X^{+}$frequencies in MSCs from H. $n=3$. (K) Quantification of the DCFDA fluorescence intensities reveals increased ROS levels in MSCs

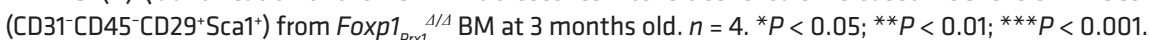

I

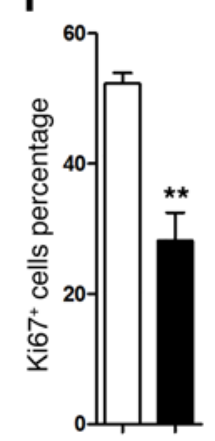

J

K
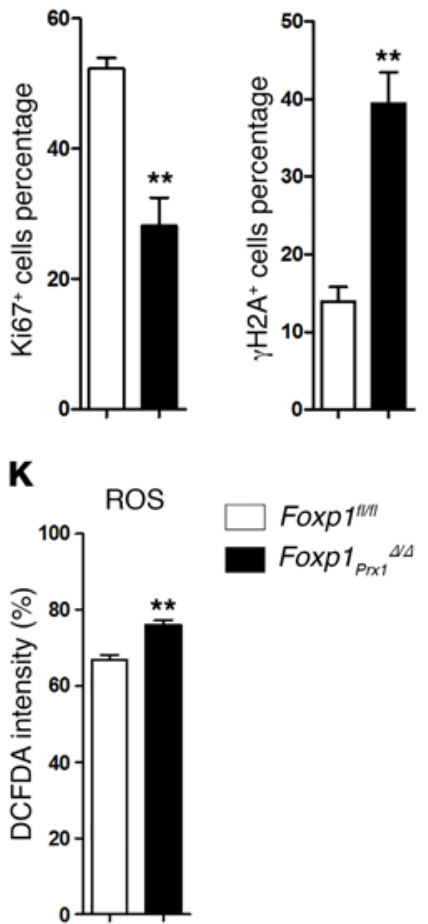
A

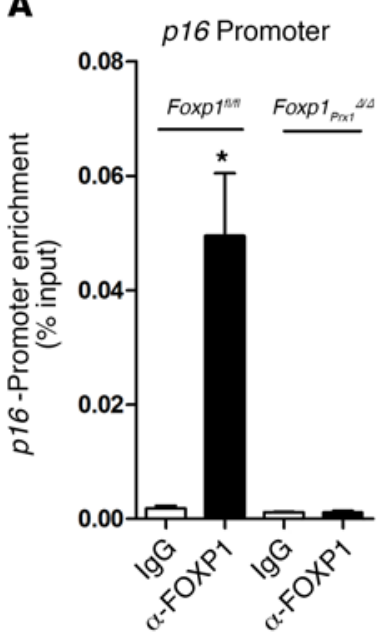

B

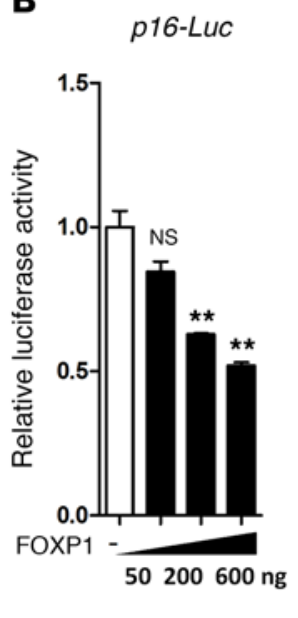

C

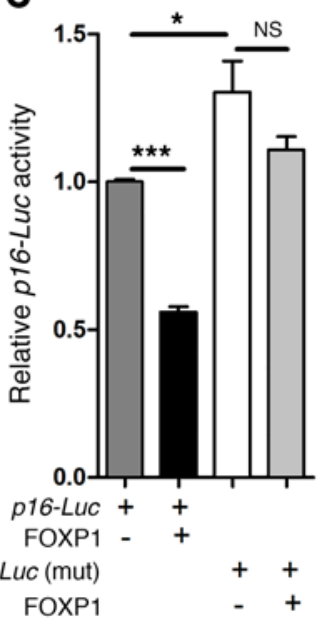

D

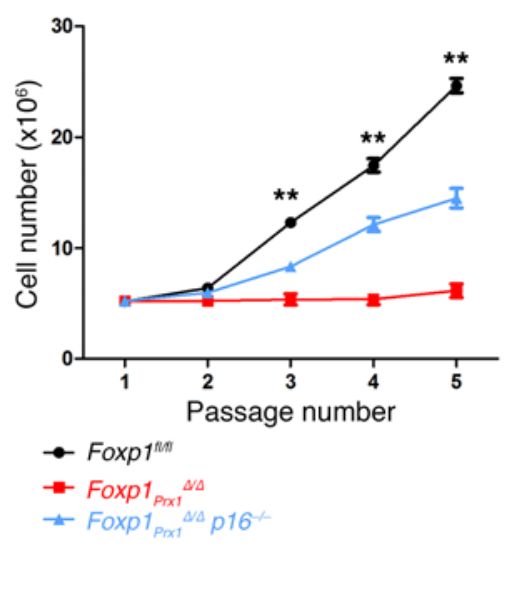

E

E Foxp $1^{\text {wn }}$ Foxp1 $1_{P x x}{ }^{\Delta \Delta}$ Foxp $1_{p \times x 1}{ }^{\Delta / \Delta} p 16^{-}$

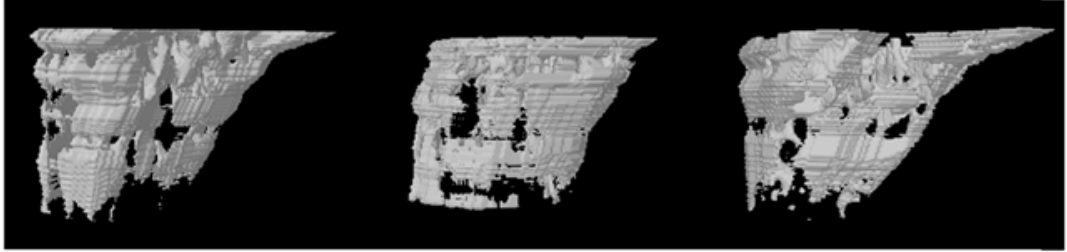

$\mathbf{F}$
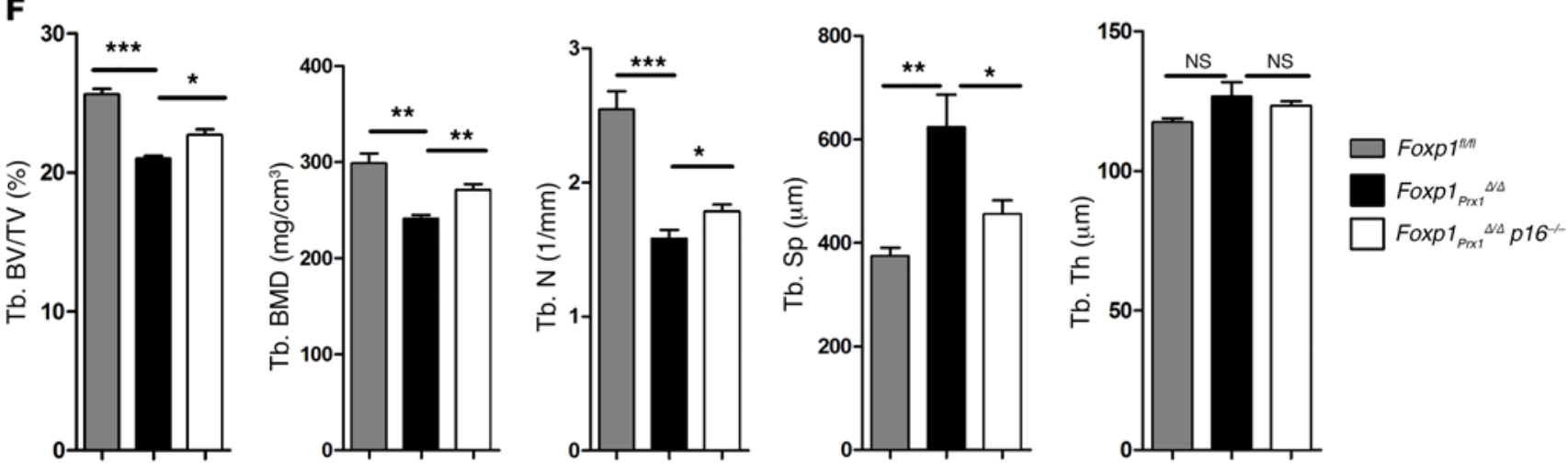

Figure 6. Foxp1 attenuates MSC senescence through repression of p16 $16^{I N 4 A}$ transcription. (A) Promoter occupancy of p16 $6^{I N 4 A}$ as assessed by anti-FOXP1 ChIP-PCR in MSCs. Sequence analysis identified a consensus FOXP1-binding site within the p16 INK4A promoter (1701-1695). (B) FOXP1 repression of p16 INK4A transcription as revealed by p16-Luc reporter assays in C3H10T1/2 cells transfected with the indicated levels of cotransfected F0XP1. (C) F0XP1 repressed transcription of p16-Luc reporter, but not the mutant reporter p16-Luc (mut). (D) Doubling times of BM MSCs expanded in vitro. The replication capacity of

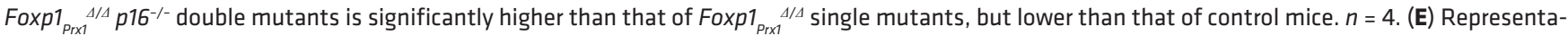

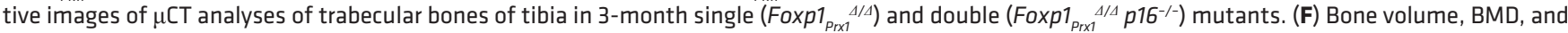

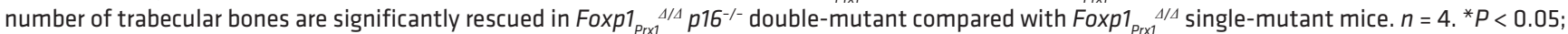
${ }^{* *} P<0.01 ;{ }^{* *} P<0.001$. Tb. BV/TV, trabecular bone volume/total volume; Tb. N, trabecular number; Tb. Sp, trabecular spacing; Tb. Th, trabecular thickness.

qPCR. Total RNA was extracted with TRIzol reagent (Invitrogen), and cDNA was generated using the GoScript Reverse Transcription System (Promega). Real-time qPCR was performed with the ABI 7500 System (Applied Biosciences) using SYBR Green (Roche). The primers used for qPCR are listed in Supplemental Table 1.

Osmium tetraoxide staining and $\mu \mathrm{CT}$ analysis. Femurs or tibias were dissected from mice and fixed in $70 \%$ ethanol at $4^{\circ} \mathrm{C}$. To visualize and quantify BM fat, bones were decalcified and stained by osmium tetraoxide as described previously (73). $\mu \mathrm{CT}$ scanning of bone and fat was performed on SkyScan 1176 (Bruker). A 3D model was reconstructed, and structural indices were calculated using
CTAn software. The region of interest (ROI) selected was $5 \mathrm{~mm}$ below the growth plate of bones.

Cell cultures. Mouse MSCs were enumerated and expanded using the mouse MesenCult Proliferation Kit (STEMCELL Technologies) according to the manufacturer's protocols. Briefly, mouse BM cells from tibia and femur were flushed out with $2 \mathrm{ml}$ IMDM with 2\% FBS and filtered through a 70- $\mu \mathrm{m}$ cell strainer (BD Falcon). Nucleated cells were counted using acetic acid with methylene blue. Cell numbers and volumes were adjusted using MesenCult medium (STEMCELL Technologies) according to assay requirements. Cell lines C3H1OT1/2 and 3T3-L1 were obtained from ATCC. 

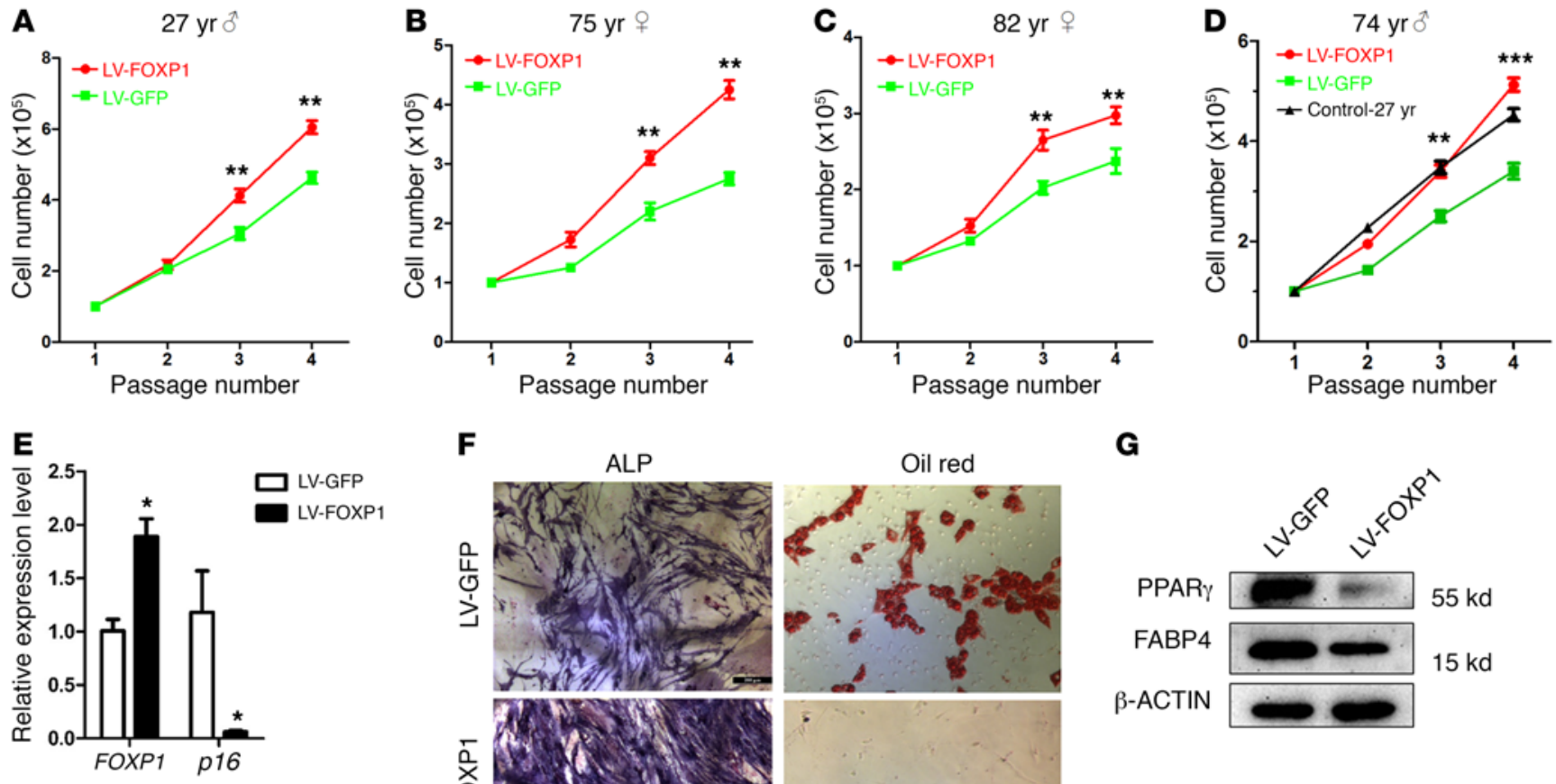

$\mathbf{F}$

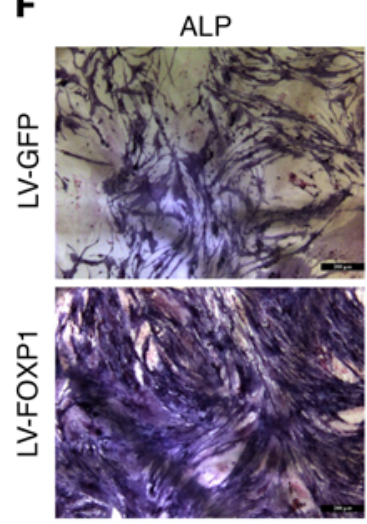

Oil red

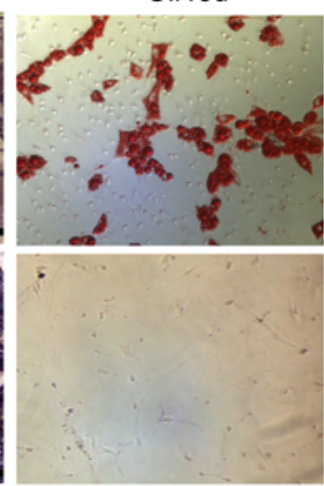

G

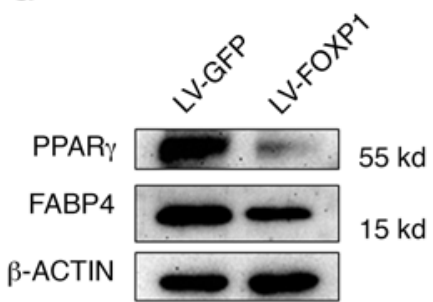

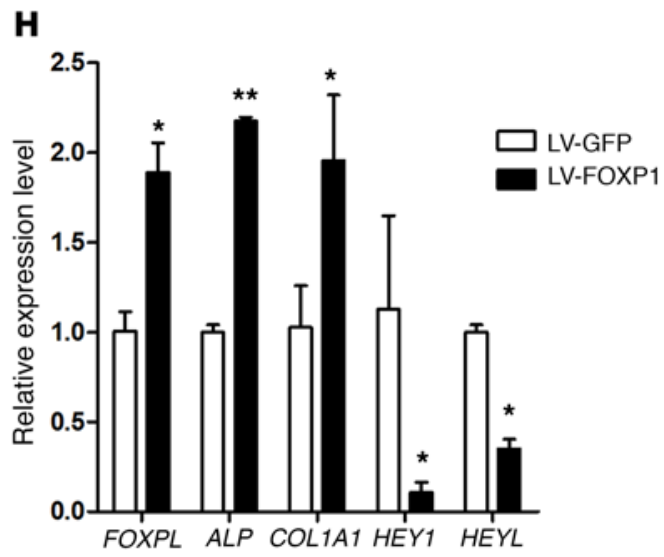

I

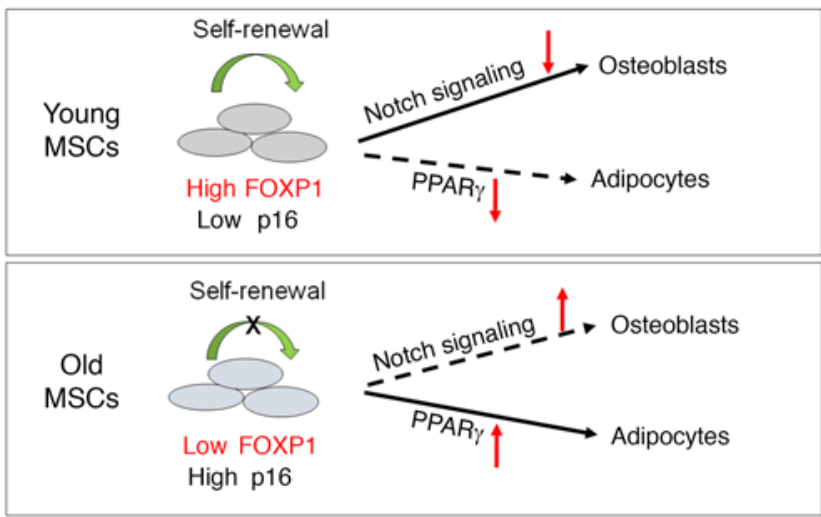

Figure 7. Overexpression of FOXP1 in hMPCs augments their expansion capacity. (A-D) In vitro expansion of hMPCs isolated from BM donors ages 27 (male, A), 75 (female, B), 82 (female, C), and 74 (male, D) years. MPCs were transfected with lentiviral LV-FOXP1 or LV-GFP. Control 27 yr, hMPCs were from a 27-year-old male donor. (E) Expression of FOXP1 and p16 INK4A in the fifth passage of hMPCs with FOXP1 overexpression from a 74-year-old donor. (F) Representative images of ALP and oil red $O$ staining 14 days after osteogenic differentiation of the 74-year-old donor's hMPCs transfected with LV-FOXP1. Scale bar: $100 \mu \mathrm{m}$. (G) Western blot for PPAR $\gamma$ and FABP4 expression in adipogenic cultures of hMPCs in F. (H) qPCR of FOXP1, ALP, COL1A1, HEY1, and HEYL expression in osteogenic cultures of FOXP1-expressing hMPCs in F. $n=3$. (I) Model by which FOXP1 regulates BM MSC aging. In young MSCs, high expression of Foxp1 restrains the activation of $p 16^{I N K 4 A}$ to maintain the replication capacity of MSCs while sustaining osteogenic rather than adipogenic differentiation. In old MSCs, reduction of Foxp1 expression releases $p 16^{I N K 4 A}$ repression, leading to impaired MSC replication capacity while promoting adipogenic differentiation at the expense of osteogenic differentiation. ${ }^{*} P<0.05 ;{ }^{* *} P<0.01 ;{ }^{* *} P<0.001$.

For mouse CFU-F assays, 2 million BM cells were plated per well in 6-well plates, each group was plated in duplicate or triplicate, and cells were cultured for 14 days at $37^{\circ} \mathrm{C}$ in $5 \% \mathrm{CO}_{2}$. CFU-F-derived colonies were stained by Giemsa staining solution and enumerated. For CFU-Ob assays, 1 million BM cells were plated on 35-mm dishes in $2 \mathrm{ml}$ complete MesenCult medium. After approximately 7 to 9 days, osteogenic medium (10 nM dexamethasone [Sigma], $10 \mathrm{mM}$ $\beta$-glycerophosphate, and $50 \mu \mathrm{M}$ ascorbic acid [Sigma] in $\alpha$-MEM
[Hyclone] containing 10\% FBS [Hyclone]) was added. At 14 days, cells were fixed and stained for ALP using TRACP \& ALP Double-Stain Kit (Clonetech) or Alizarin red S. For CFU-Ad assays, 2 million BM cells were plated per well in 24-well plates with $0.5 \mathrm{ml}$ Complete MesenCult Medium (STEMCELL Technologies). Culture conditions were changed to adipogenic medium (DMEM supplemented with $10 \% \mathrm{FBS}$, $1 \mu \mathrm{M}$ dexamethasone, $10 \mu \mathrm{g} / \mathrm{ml}$ insulin, $0.5 \mathrm{mM}$ 3-isobutyl-1-methylxanthine, and $200 \mu \mathrm{M}$ indomethacin) when cells reached $80 \%$ to $90 \%$ 
confluence. Cells were cultured for 1 week before oil red O staining. Oil red O staining was performed according to standard protocols. For population doubling assays, the first passaged mouse MSCs were seeded in $10-\mathrm{cm}$ plates with $5 \times 10^{6}$ cells and hMPCs were seeded in 35 -mm dishes with $1 \times 10^{5}$ cells. Medium changes were performed every 3 days and cells passaged at weekly intervals. Cells were counted at each passage to calculate population doublings.

hMPCs were isolated from vertebrae or femur bones of trauma patients between 20 and 80 years old. hMPCs were then separated using the Human Marrow Lymphocyte Medium Kit (Sangon Biotech) and cultured using the MesenCult Proliferation Kit (human) (STEMCELL Technologies). For osteogenic differentiation of human mesenchymal stem cells, 0.1 million hMPCs were seeded per well of 24-well plates with the same medium for CFU-Ob assays above. After 9 days of induction, cells were fixed and stained for ALP.

Viral infection. To obtain transformants stably expressing FOXP1, pMSCV-FOXP1-puro and pMSCV-GFP-puro control retroviruses were transfected into Plat-E packaging cells using FuGENE HD (Promega). After 48 hours, culture supernatants were collected and used for infection. Retroviruses were introduced into $\mathrm{C} 3 \mathrm{H} 10 \mathrm{~T} 1 / 2$ cells, and stable transformants were selected with $5 \mu \mathrm{g} / \mathrm{ml}$ puromycin. For osteoblast and adipocyte differentiation, cells were cultured in osteogenic medium and adipogenic medium described above, respectively. For lentivirus infections, hMPCs were plated at a density of $1 \times 10^{5}$ cells in $3.5-\mathrm{mm}$ dishes. LV-FOXP1 or LV-GFP virus was added at a multiplicity of infection of 20, and cells were exposed to virus for 24 hours and selected with $1 \mu \mathrm{g} / \mathrm{ml}$ puromycin for 3 days. Lentiviruses were obtained from Hanbio.

ChIP assay. Briefly, after MSCs were isolated from mouse BM and cultured for 2 weeks, chromatin was crosslinked with $1 \%$ formaldehyde and sheared to 300- to 500-bp fragments by sonication (Sonics VCX130, 30\% amplitude, 5 seconds on and 10 seconds off for 16 cycles). FOXP1 antibody (Millipore, ABE68, 1:200) and normal rabbit IgG (Santa Cruz Biotechnology Inc., sc-2027, 1:80) were used to immunoprecipitate the relevant protein-DNA complex. The crosslinked DNA and protein were reversed by addition of $5 \mathrm{M} \mathrm{NaCl}$; DNA was extracted by phenol/chloroform and precipitated with ethanol and glycogen. Purified DNA was quantified using real-time PCR. The primer sequences are listed in Supplemental Table 1.

Luciferase reporter assay. Luciferase assays were performed in HEK293T, C3H10T1/2, or 3T3-L1 cells. The reporter plasmid PpargLuc, containing a 2.2-kb fragment of the 5 ' flanking region of the Pparg gene, was obtained from Hiroshi Takayanagi (Tokyo Medical and Dental University, Tokyo, Japan). The RBP-JK luciferase reporter plasmid and expression plasmid with NICD2 were provided by Lianping Xing (University of Rochester Medical Center, Rochester, New York, USA). The $p 16^{I N K 4 A}$-luc reporter plasmid, which contains a $2.8-\mathrm{kb}$ fragment of the $5^{\prime}$ flanking region of the $p 16^{I N K 4 A}$ gene, was constructed in our lab. The mutant $p 16^{I N K 4 A}$-luc reporter plasmid was generated by replacing the putative FOXP1-binding site (TATTTAT) at position -1701 with the random sequence GCGGGCG. The expression plasmids of FOXP1,

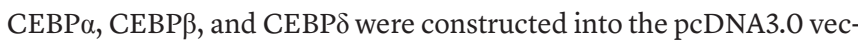
tor. Cells were transfected using Lipofectamine 2000 (Invitrogen) in 24 -well plates. The transfection amount of each plasmid was $200 \mu \mathrm{g}$, and the total amount of transfected DNA was kept consistent across each transfection condition of pcDNA3.0 empty plasmids where necessary. After 32 hours, dual luciferase assay was performed according to the manufacturer's protocols (Promega).
co-IP. For in vitro co-IP, His- or FLAG-tagged proteins were produced in HEK293T cells transfected by Lipofectamine 2000 with corresponding plasmids. For in vivo co-IP, BM MSCs were isolated and cultured from WT mice. Total cell lysates were incubated overnight at $4^{\circ} \mathrm{C}$ with antibodies (described below) or normal IgG (Santa Cruz Biotechnology Inc., sc-2027) as control. Antibody-antigen complexes were precleared with Protein A/G PLUS-Agarose (Santa Cruz Biotechnology Inc., sc-2003). After several washes, samples were boiled and analyzed by immunoblot.

Western blot. Cells were lysed by NP40 lysis buffer (Beyotime Biotechnology) supplemented with protease inhibitor and phosphatase inhibitor cocktails (Selleck). Protein samples were separated by SDS-PAGE, transferred to a nitrocellulose filter membrane (BioRad), blocked with 5\% nonfat milk (in TBST), and incubated with primary antibodies against FOXP1 (Millipore, ABE68, 1:1000), C/EBP $\beta$ (Santa Cruz Biotechnology Inc., sc-150, 1:500), C/EBP $\delta$ (Santa Cruz Biotechnology Inc., sc-151, 1:500), FABP4 (Abcam, ab13979, 1:1000), RBPjк (Santa Cruz Biotechnology Inc., sc-28713, 1:500), $\gamma$ H2A antibody (GeneTex, GTX11174, 1:1000), Bmi (Abcam, ab14389, 1:1000), PPAR $\gamma$ (Santa Cruz Biotechnology Inc., sc-22020, 1:500), p16 ${ }^{\text {INK4a }}$ (Millipore, 04-239, 1:1000), H3K9me3 (Abcam, ab8898, 1:1000), LAP2 $\beta$ (BD, 611000, 1:1000), His-Tag (MBL, M136-3, 1:2000), FLAG (Agilent, 200471, 1:2000), or $\beta$-actin (Selleck, A1016, 1:2000) at $4^{\circ} \mathrm{C}$ overnight. Proteins were visualized using horseradish peroxidase-conjugated (HRP-conjugated) secondary antibody and chemiluminescent HRP substrate (Millipore).

Immunohistochemistry and confocal microscope. Cultured MPCs were fixed in $4 \%$ PFA for 10 minutes at room temperature. After blocking with $10 \%$ normal goat serum, cells were then incubated with $\gamma \mathrm{H} 2 \mathrm{~A}$ antibody (GeneTex, GTX11174, 1:500), Ki67 antibody (Abcam, ab15580, 1:500), and LAP2 $\beta$ (BD, 611000, 1:500), followed by incubation with Alexa Fluor 488 - or Alexa Fluor 594-conjugated secondary antibodies. For SA- $\beta$-gal staining, cells were fixed with $0.5 \%$ glutaraldehyde in $\mathrm{PBS}$ ( $\mathrm{pH}$ 7.2) for 5 minutes at room temperature, then washed with PBS containing $\mathrm{MgCl}_{2}$ $\left(\mathrm{pH} 7.2,1 \mathrm{mM} \mathrm{MgCl}_{2}\right.$ ) and stained with X-gal solution ( $1 \mathrm{mg} / \mathrm{ml} \mathrm{X-gal,} 0.12$ $\mathrm{mM} \mathrm{K}_{3} \mathrm{Fe}[\mathrm{CN}]_{6}, 1 \mathrm{mM} \mathrm{MgCl}_{2}$ in PBS, pH 6.0) overnight at $37^{\circ} \mathrm{C}$.

Bones were fixed in $4 \%$ PFA for 24 hours at $4^{\circ} \mathrm{C}$, decalcified in $10 \%$ EDTA ( $\mathrm{pH}$ 7.4) for 21 days, embedded in paraffin or tissue-freezing medium (Leica), and sectioned to $8 \mu \mathrm{m}$. For immunofluorescence, heat-induced antigen retrieval with sodium citrate buffer $(10 \mathrm{mM}$ sodium citrate, $0.05 \%$ Tween 20 , pH 6.0) was performed before bone sections were blocked with $10 \%$ normal serum containing $1 \%$ BSA in TBST ( $\mathrm{pH}$ 7.6) for 2 hours at room temperature, then incubated overnight at $4^{\circ} \mathrm{C}$ with primary antibodies to mouse FOXP1 (Millipore, ABE68,1:100) and Nestin (Abcam, ab11306, 1:100) diluted in TBST with 1\% BSA. Subsequently, sections were incubated with secondary fluorescentconjugated antibodies at room temperature for 2 hours in the dark. Samples were imaged by the Leica TCS SP5 confocal microscope, the Leica DM2500, or the $3000 B$ microscope. H\&E staining was conducted according to standard protocols.

Flow cytometry analysis. For BrdU labeling of MSCs, mice were intraperitoneally injected with BrdU $(100 \mu \mathrm{g} / \mathrm{g}$ of body mass, SigmaAldrich) and sacrificed 48 hours later. BM cells were flushed out and collected in DMEM with $2 \% \mathrm{FBS}$, then diluted to 8 million cells in $400 \mu \mathrm{l}$ of medium and incubated with anti-mouse CD45-APC (17-0451, eBioscience), CD11b-APC (17-0112, eBioscience), Ter119-APC (17-5921, eBioscience), CD31-APC (17-0311, eBioscience), Sca1-PerCP (45-5981, 
eBioscience), and CD29-PE antibodies (12-0291, eBioscience) in the dark for 30 minutes at $4{ }^{\circ} \mathrm{C}$. After surface staining, cells were fixed with $1 \%$ PFA and permeabilized with $0.5 \%$ Tween-20. DNA was fragmented by DNase I; then cells were stained with anti-BrdU-FITC (11-5071, eBioscience) antibody or DCFDA for 45 minutes at room temperature. Cells were acquired on a BD FACSCalibur, and analysis was performed with FlowJo software, version 7.6. For MSC sorting, BM cells were stained with CD45-APC, Ter119-APC, CD31-APC, Sca1-PerCP, and CD29-PE antibodies and sorted on a BD FACSAria II.

Genomic DNA isolation and bisulfite sequencing PCR. MSC genomic DNA was extracted with the TIANamp genomic DNA kit (TIANGEN), and bisulfite sequencing PCR was performed with Oebiotech reagents. The sequencing primers were 5'-ATAGTAATTAAAGAGGAGTTGTTGGGG-3' and 5'-CCTAACACTCTCCATATAACCRC-3'.

Statistics. All data are presented as mean \pm SEM. Error bars indicate SEM. Nonlinear regression analysis with exponential 2-phase decay was used for Foxp1 and p16 expression. Two-tailed Student's $t$ tests were used for comparisons between 2 groups, and 1-way ANOVA with Bonferroni's post-hoc analysis was used for multiple comparisons (3 or more groups). $P<0.05$ was considered significant.

Study approval. All murine studies were conducted under the approved Shanghai Jiao Tong University IACUC protocol SYXK 20110112. For all human studies, written informed consent was received from participants prior to inclusion in the study where required. All samples were obtained in accordance with standard protocols of the Bioethics Committee of Bio-X Institutes at Shanghai Jiao Tong University.

\section{Author contributions}

HL, PL, SX, ZY, and XG designed and performed experiments. YL, $\mathrm{ZZ}, \mathrm{YH}, \mathrm{GY}, \mathrm{TT}, \mathrm{YF}$, and YR provided human biospecimens. BL, JDD, and HOT provided mouse lines. XG wrote the manuscript.

\section{Acknowledgments}

This work was supported by research funding from the National Major Fundamental Research 973 Program of China (2014CB942902 and 2012CB966903) and grants from the National Natural Science Foundation of China (31171396, $31271553,81421061,31100624$, and 81200586 to XG), the NIH (R01CA31534), the Cancer Prevention Research Institute of Texas (RP120348 and RP120459), the Marie Betzner Morrow Centennial Endowment (to HOT), and the Lymphoma Research Foundation (300463 to JDD).

Address correspondence to: Xizhi Guo, 800 Dongchuan Road, Biomedical Building \#1-205, Shanghai, China, 200240. Phone: 86.021.34206736; E-mail: xzguo2005@sjtu.edu.cn.
1. Verma S, Rajaratnam JH, Denton J, Hoyland JA, Byers RJ. Adipocytic proportion of bone marrow is inversely related to bone formation in osteoporosis. JClin Pathol. 2002;55(9):693-698.

2. Demontiero O, Vidal C, Duque G. Aging and bone loss: new insights for the clinician. Ther $A d v$ Musculoskelet Dis. 2012;4(2):61-76.

3. Chan GK, Duque G. Age-related bone loss: old bone, new facts. Gerontology. 2002;48(2):62-71.

4. Stenderup K, Justesen J, Clausen C, Kassem M. Aging is associated with decreased maximal life span and accelerated senescence of bone marrow stromal cells. Bone. 2003;33(6):919-926.

5. Méndez-Ferrer S, et al. Mesenchymal and haematopoietic stem cells form a unique bone marrow niche. Nature. 2010;466(7308):829-834.

6. Ding L, Morrison SJ. Haematopoietic stem cells and early lymphoid progenitors occupy distinct bone marrow niches. Nature. 2013;495(7440):231-235.

7. Zhou BO, Yue R, Murphy MM, Peyer JG, Morrison SJ. Leptin-receptor-expressing mesenchymal stromal cells represent the main source of bone formed by adult bone marrow. Cell Stem Cell. 2014;15(2):154-168.

8. Rosen CJ, Ackert-Bicknell C, Rodriguez JP, Pino AM. Marrow fat and the bone microenvironment: developmental, functional, and pathological implications. Crit Rev Eukaryot Gene Expr. 2009;19(2):109-124.

9. Chen Q, et al. Fate decision of mesenchymal stem cells: adipocytes or osteoblasts? Cell Death Differ. 2016;23(7):1128-1139.

10. Farmer SR. Transcriptional control of adipocyte formation. Cell Metab. 2006;4(4):263-273.

11. Ducy P, Zhang R, Geoffroy V, Ridall AL, Karsenty G. Osf2/Cbfa1: a transcriptional activator of osteoblast differentiation. Cell.
1997;89(5):747-754.

12. Nakashima K, et al. The novel zinc fingercontaining transcription factor osterix is required for osteoblast differentiation and bone formation. Cell. 2002;108(1):17-29.

13. Serrano M, Lin AW, McCurrach ME, Beach D, Lowe SW. Oncogenic ras provokes premature cell senescence associated with accumulation of p53 and p16INK4a. Cell. 1997;88(5):593-602.

14. Collado M, Blasco MA, Serrano M. Cellular senescence in cancer and aging. Cell. 2007;130(2):223-233.

15. Krishnamurthy J, et al. p16INK4a induces an age-dependent decline in islet regenerative potential. Nature. 2006;443(7110):453-457.

16. Molofsky AV, et al. Increasing p16INK4a expression decreases forebrain progenitors and neurogenesis during ageing. Nature. 2006;443(7110):448-452.

17. Janzen $\mathrm{V}$, et al. Stem-cell ageing modified by the cyclin-dependent kinase inhibitor p16INK4a. Nature. 2006;443(7110):421-426.

18. Krishnamurthy J, et al. Ink4a/Arf expression is a biomarker of aging. J Clin Invest. 2004;114(9):1299-1307.

19. Gu Z, et al. Upregulation of p16INK4A promotes cellular senescence of bone marrow-derived mesenchymal stem cells from systemic lupus erythematosus patients. Cell Signal. 2012;24(12):2307-2314.

20. Tyner SD, et al. p53 mutant mice that display early ageing-associated phenotypes. Nature. 2002;415(6867):45-53.

21. Jacobs JJ, Kieboom K, Marino S, DePinho RA, van Lohuizen M. The oncogene and Polycomb-group gene bmi-1 regulates cell proliferation and senescence through the ink 4 a locus. Nature. 1999;397(6715):164-168.
22. Cao L, Li W, Kim S, Brodie SG, Deng CX. Senescence, aging, and malignant transformation mediated by $\mathrm{p} 53$ in mice lacking the Brca1 fulllength isoform. Genes Dev. 2003;17(2):201-213.

23. Varela I, et al. Accelerated ageing in mice deficient in Zmpste24 protease is linked to 533 signalling activation. Nature. 2005;437(7058):564-568.

24. Nishino J, Kim I, Chada K, Morrison SJ. Hmga2 promotes neural stem cell self-renewal in young but not old mice by reducing p16Ink4a and p19Arf Expression. Cell. 2008;135(2):227-239.

25. Baker DJ, et al. Clearance of p16Ink4a-positive senescent cells delays ageing-associated disorders. Nature. 2011;479(7372):232-236.

26. Lin SP, Chiu FY, Wang Y, Yen ML, Kao SY, Hung SC. RB maintains quiescence and prevents premature senescence through upregulation of DNMT1 in mesenchymal stromal cells. Stem Cell Reports. 2014;3(6):975-986.

27. Calo E, Quintero-Estades JA, Danielian PS, Nedelcu S, Berman SD, Lees JA. Rb regulates fate choice and lineage commitment in vivo. Nature. 2010;466(7310):1110-1114.

28. Gabut M, et al. An alternative splicing switch regulates embryonic stem cell pluripotency and reprogramming. Cell. 2011;147(1):132-146.

29. $\mathrm{Hu} \mathrm{H}$, et al. Foxp1 is an essential transcriptional regulator of B cell development. Nat Immunol. 2006;7(8):819-826.

30. Feng X, Wang H, Takata H, Day TJ, Willen J, Hu H. Transcription factor Foxp1 exerts essential cell-intrinsic regulation of the quiescence of naive T cells. Nat Immunol. 2011;12(6):544-550.

31. Feng $X$, et al. Foxp1 is an essential transcriptional regulator for the generation of quiescent naive T cells during thymocyte development. Blood. 2010;115(3):510-518. 
32. Li S, et al. Foxp1/4 control epithelial cell fate during lung development and regeneration through regulation of anterior gradient 2. Development. 2012;139(14):2500-2509.

33. Wang B, et al. Foxp1 regulates cardiac outflow tract, endocardial cushion morphogenesis and myocyte proliferation and maturation. Development. 2004;131(18):4477-4487.

34. Shu W, Lu MM, Zhang Y, Tucker PW, Zhou D, Morrisey EE. Foxp2 and Foxp1 cooperatively regulate lung and esophagus development. Development. 2007;134(10):1991-2000.

35. Li S, et al. Epithelium-generated neuropeptide $\mathrm{Y}$ induces smooth muscle contraction to promote airway hyperresponsiveness. J Clin Invest. 2016;126(5):1978-1982.

36. Zhang Y, et al. Foxp1 coordinates cardiomyocyte proliferation through both cell-autonomous and nonautonomous mechanisms. Genes Dev. 2010;24(16):1746-1757.

37. Leishman E, et al. Foxp1 maintains hair follicle stem cell quiescence through regulation of Fgf18 Development. 2013;140(18):3809-3818.

38. Araujo DJ, et al. FoxP1 orchestration of ASDrelevant signaling pathways in the striatum. Genes Dev. 2015;29(20):2081-2096.

39. Adams KL, Rousso DL, Umbach JA, Novitch BG. Foxp1-mediated programming of limbinnervating motor neurons from mouse and human embryonic stem cells. Nat Commun. 2015;6:6778.

40. Zou Y, et al. Forkhead box P1 (FOXP1) transcription factor regulates hepatic glucose homeostasis. J Biol Chem. 2015;290(51):30607-30615.

41. Carr CW, et al. Chiari I malformation, delayed gross motor skills, severe speech delay, and epileptiform discharges in a child with FOXP1 haploinsufficiency. Eur J Hum Genet. 2010;18(11):1216-1220.

42. Wang B, Lin D, Li C, Tucker P. Multiple domains define the expression and regulatory properties of Foxp1 forkhead transcriptional repressors. J Biol Chem. 2003;278(27):24259-24268.

43. Chamberlain AA, et al. DNA methylation is developmentally regulated for genes essential for cardiogenesis. J Am Heart Assoc. 2014;3(3):e000976.

44. Greenbaum A, et al. CXCL12 in early mesenchymal progenitors is required for haematopoietic stem-cell maintenance. Nature. 2013;495(7440):227-230 .

45. Nakachi $Y$, et al. Identification of novel PPARgamma target genes by integrated analysis of ChIPon-chip and microarray expression data during adipocyte differentiation. Biochem Biophys Res Commun. 2008;372(2):362-366.

46. Zhong ZA, Zahatnansky J, Snider J, Van Wieren E, Diegel CR, Williams BO. Wntless spatially regulates bone development through $\beta$-catenindependent and independent mechanisms. Dev Dyn. 2015;244(10):1347-1355.

47. Brun RP, Kim JB, Hu E, Altiok S, Spiegelman BM. Adipocyte differentiation: a transcriptional regulatory cascade. Curr Opin Cell Biol. 1996;8(6):826-832.

48. Hilton MJ, et al. Notch signaling maintains bone marrow mesenchymal progenitors by suppressing osteoblast differentiation. Nat Med. 2008;14(3):306-314.

49. Tao J, Chen S, Lee B. Alteration of Notch signaling in skeletal development and disease. Ann N Y Acad Sci. 2010;1192:257-268.

50. Zhang W, et al. Aging stem cells. A Werner syndrome stem cell model unveils heterochromatin alterations as a driver of human aging. Science. 2015;348(6239):1160-1163.

51. Kim WY, Sharpless NE. The regulation of INK4/ARF in cancer and aging. Cell. 2006;127(2):265-275.

52. Kua HY, et al. c-Abl promotes osteoblast expansion by differentially regulating canonical and non-canonical BMP pathways and p16INK4a expression. Nat Cell Biol. 2012;14(7):727-737.

53. Kubo H, et al. Identification of mesenchymal stem cell (MSC)-transcription factors by microarray and knockdown analyses, and signature molecule-marked MSC in bone marrow by immunohistochemistry. Genes Cells. 2009;14(3):407-424

54. Benisch P, et al. The transcriptional profile of mesenchymal stem cell populations in primary osteoporosis is distinct and shows overexpression of osteogenic inhibitors. PLOS ONE. 2012;7(9):e45142.

55. Jones MJ, Goodman SJ, Kobor MS. DNA methylation and healthy human aging. Aging Cell. 2015;14(6):924-932.

56. Moerman EJ, Teng K, Lipschitz DA, LeckaCzernik B. Aging activates adipogenic and suppresses osteogenic programs in mesenchymal marrow stroma/stem cells: the role of PPAR-gamma2 transcription factor and TGF-beta/BMP signaling pathways. Aging Cell. 2004;3(6):379-389.

57. Akune T, et al. PPARgamma insufficiency enhances osteogenesis through osteoblast formation from bone marrow progenitors. J Clin Invest. 2004;113(6):846-855.

58. Ge C, Cawthorn WP, Li Y, Zhao G, Macdougald OA, Franceschi RT. Reciprocal control of osteogenic and adipogenic differentiation by ERK/MAP kinase phosphorylation of Runx2 and PPAR $\gamma$ transcription factors. J Cell Physiol. 2016;231(3):587-596.

59. Sun H, Kim JK, Mortensen R, Mutyaba LP, Hankenson KD, Krebsbach PH. Osteoblast-targeted suppression of PPAR $\gamma$ increases osteogenesis through activation of mTOR signaling. Stem
Cells. 2013;31(10):2183-2192.

60. Zhao H, et al. Foxp1/2/4 regulate endochondral ossification as a suppresser complex. Dev Biol. 2015;398(2):242-254

61. Marie PJ, Kassem M. Osteoblasts in osteoporosis: past, emerging, and future anabolic targets. Eur J Endocrinol. 2011;165(1):1-10.

62. Banham AH, et al. The FOXP1 winged helix transcription factor is a novel candidate tumor suppressor gene on chromosome 3p. Cancer Res. 2001;61(24):8820-8829.

63. Wlodarska I, et al. FOXP1, a gene highly expressed in a subset of diffuse large B-cell lymphoma, is recurrently targeted by genomic aberrations. Leukemia. 2005;19(8):1299-1305.

64. Fenton JA, et al. $\mathrm{t}(3 ; 14)(\mathrm{p} 14 ; \mathrm{q} 32)$ results in aberrant expression of FOXP1 in a case of diffuse large B-cell lymphoma. Genes Chromosomes Cancer. 2006;45(2):164-168.

65. Brown P, Marafioti T, Kusec R, Banham AH. The FOXP1 transcription factor is expressed in the majority of follicular lymphomas but is rarely expressed in classical and lymphocyte predominant Hodgkin's lymphoma. J Mol Histol. 2005;36(4):249-256.

66. Put N, Deeren D, Michaux L, Vandenberghe P. FOXP1 and PAX5 are rare but recurrent translocations partners in acute lymphoblastic leukemia. Cancer Genet. 2011;204(8):462-464.

67. Shigekawa T, et al. FOXP1, an estrogen-inducible transcription factor, modulates cell proliferation in breast cancer cells and 5-year recurrence-free survival of patients with tamoxifen-treated breast cancer. Horm Cancer. 2011;2(5):286-297.

68. Toma MI, et al. Expression of the Forkhead transcription factor FOXP1 is associated with tumor grade and Ki67 expression in clear cell renal cell carcinoma. Cancer Invest. 2011;29(2):123-129.

69. Hu CR, Wang JH, Wang R, Sun Q, Chen LB. Both FOXP1 and p65 expression are adverse risk factors in diffuse large $\mathrm{B}$-cell lymphoma: a retrospective study in China. Acta Histochem. 2013;115(2):137-143.

70. Ijichi N, Ikeda K, Horie-Inoue K, Inoue S. FOXP1 and estrogen signaling in breast cancer. Vitam Horm. 2013;93:203-212.

71. Dekker JD, et al. Subtype-specific addiction of the activated B-cell subset of diffuse large B-cell lymphoma to FOXP1. Proc Natl Acad Sci USA. 2016;113(5):E577-E586.

72. Logan M, Martin JF, Nagy A, Lobe C, Olson EN, Tabin CJ. Expression of Cre Recombinase in the developing mouse limb bud driven by a Prxl enhancer. Genesis. 2002;33(2):77-80.

73. Scheller EL, et al. Use of osmium tetroxide staining with microcomputerized tomography to visualize and quantify bone marrow adipose tissue in vivo. Meth Enzymol. 2014;537:123-139. 